\title{
Blood flow velocity field estimation via spatial regression with PDE penalization
}

\author{
Laura Azzimonti Laura M. Sangalli* Piercesare Secchi Maurizio Domanin \\ Fabio Nobile
}

\begin{abstract}
We propose an innovative method for the accurate estimation of surfaces and spatial fields when a prior knowledge on the phenomenon under study is available. The prior knowledge included in the model derives from physics, physiology or mechanics of the problem at hand, and is formalized in terms of a partial differential equation governing the phenomenon behavior, as well as conditions that the phenomenon

\footnotetext{
*Laura Azzimonti is Post-Doctoral Fellow, Laura M. Sangalli is Assistant Professor and Piercesare Secchi is Professor, MOX - Dipartimento di Matematica, Politecnico di Milano, Piazza Leonardo da Vinci 32, 20133 Milano, Italy. Maurizio Domanin is Professor, U.O. di Chirurgia Vascolare Fondazione I.R.C.C.S. Ca' Granda Ospedale Maggiore Policlinico, Milano, and Università degli Studi di Milano. Fabio Nobile is Professor, Mathematics Institute of Computational Science and Engineering, École Polytechnique Fédérale de Lausanne, Switzerland. The authors are grateful to James Ramsay for his advise on this work, to Christian Vergara - P.I. of the MACAREN@MOX project, to Elena Faggiano for the reconstruction of carotid geometry from MRI data, and to Silvia Romagnoli for the acquisition of eco-doppler data. The authors would also like to thank the Associate Editor and three anonymous reviewers for their constructive comments. This work was supported by the program Dote Ricercatore Politecnico di Milano - Regione Lombardia, research project "Functional data analysis for life sciences”, and by MIUR Ministero dell'Istruzione dell'Università e della Ricerca, starting grant project FIRB Futuro in Ricerca "Advanced statistical and numerical methods for the analysis of high dimensional functional data in life sciences and engineering", http://mox.polimi.it/users/sangalli/firbSNAPLE.html, P.I.: Laura M. Sangalli. Correspondence on this work should be sent to Laura M. Sangalli (E-mail: laura.sangalli@ polimi.it).
}

has to satisfy at the boundary of the problem domain. The proposed models exploit advanced scientific computing techniques and specifically make use of the Finite Element method. The estimators have a penalized regression form and the usual inferential tools are derived. Both the pointwise and the areal data frameworks are considered. The driving application concerns the estimation of the blood-flow velocity field in a section of a carotid artery, using data provided by echo-color doppler. This applied problem arises within a research project that aims at studying atherosclerosis pathogenesis.

Keywords: functional data analysis, spatial data analysis, object-oriented data analysis, penalized regression, Finite Elements.

\section{Introduction}

In this work we propose a non-parametric regression technique for surface and spatial field estimation, able to include prior knowledge on the shape of the surface or spatial field and to comply with complex conditions at the boundary of the problem domain. The motivating applied problem concerns the estimation of the blood-flow velocity field on a cross-section of an artery, using data provided by echo-color doppler acquisitions. This study is carried 
out within the project MAthematics for CARotid ENdarterectomy@MOX ${ }^{\dagger}$, that gathers researchers in statistics, numerical analysis and computer sciences and medical doctors in cardiac surgery, with the aim of investigating the pathogenesis of atherosclerosis in human carotids. The project intends specifically to study the role of blood fluid-dynamics and vessel morphology on the formation process and histological properties of atherosclerotic plaques. Interactions between the hemodynamics and atherosclerotic plaques have been highlighted for instance in Moyle et al. (2006) via numerical simulations of the blood flow on real patient-specific vessel morphologies.

The data collected within the project include: Echo-Color Doppler (ECD) measurements of blood flow at a cross-section of the common carotid artery, $2 \mathrm{~cm}$ before the carotid bifurcation, for patients affected by high grade stenosis $(>70 \%)$ in the internal carotid artery; the reconstruction of the shape of this cross-section obtained via segmentation of Magnetic Resonance Imaging (MRI) data. The first phase of the project requires the estimation, starting from these data, of the blood-flow velocity fields in the considered carotid section. These estimates are first of all of interest to the medical doctors, as they highlight relevant features of the blood flow, such as the eccentricity and the asymmetry of the flow or the reversion of the fluxes, which could have an impact on the pathology. Moreover, they will enable a population study that explores quantitatively the relation-

\footnotetext{
${ }^{\dagger}$ The MACAREN@MOX project involves Ca' Granda Ospedale Maggiore Policlinico - Milano, the MOX Laboratory for Modeling and Scientific Computing - Dipartimento di Matematica - Politecnico di Milano, the Dipartimento di Ingegneria Università degli studi di Bergamo, and the Mathematics Institute of Computational Science and Engineering - École Polytechnique Fédérale de Lausanne.
}

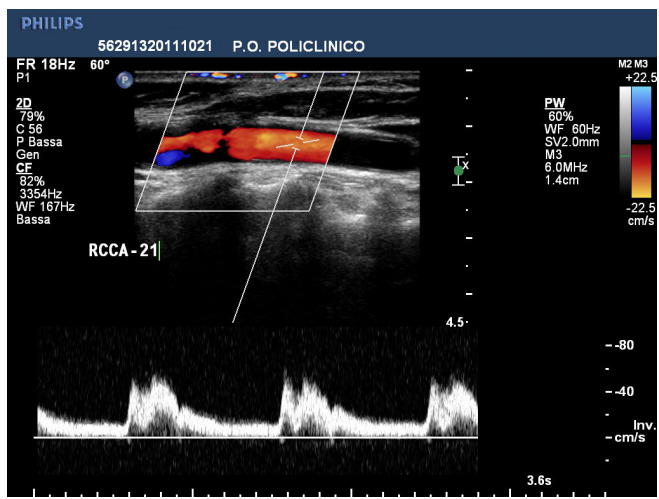

Figure 1: ECD image corresponding to the central point of the carotid section located $2 \mathrm{~cm}$ before the carotid bifurcation.

ship between the blood-flow and the atherosclerosis. Finally, the estimated blood velocity fields will also be used as patient-specific and physiological inflow conditions for hemodynamics simulations, that in turns aim at further enhancing the knowledge on this relationship. Analogous problems in hemodynamics are described in D'Elia et al. (2012), D'Elia and Veneziani (2012) and in Rozza et al. (2012).

Carotid Echo-Color Doppler (ECD) is a medical imaging procedure that uses reflected ultrasound waves to create images of an artery and to measure the velocity of blood cells in some locations within the artery. This technique does not require the use of contrast media or ionizing radiation and has relative low cost. Thanks to this complete non-invasivity and also to the short acquisition time required, ECD scans are largely used in clinics, even though they provide a less rich and noisier information than other diagnostic devices, such as Phase Contrast Magnetic Resonance Imaging. Figure 1 shows one of the ECD images used in the study. The ultrasound image in the upper part of the figure represents the longitudinal section of the vessel. It also shows by a small 


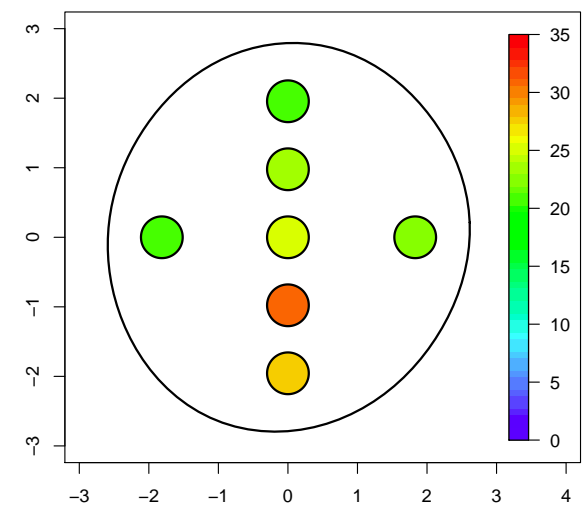

Figure 2: MRI reconstruction of the cross-section of the carotid artery located $2 \mathrm{~cm}$ before the carotid bifurcation; cross-shaped pattern of observations with each beam colored according to the mean blood-velocity measured on the beam at systolic peak time.

gray box the position of the beam where blood particle velocities, in the longitudinal direction of the vessel, are measured; the dimension of the box relates to the dimension of the beam. In the case considered in this picture, the acquisition beam is located in the center of the considered cross-section of the artery. The lower part of the ECD image is a graphical display of the acquired velocity signal during the time lapse of about three heart beats. This signal represents the histogram of the measured velocities, evolving in time. More precisely, the $\mathrm{x}$-axis represents time and the y-axis represents velocity classes; for any fixed time, the gray-scaled intensity of pixels is proportional to the number of blood-cells in the beam moving at a certain velocity. For the purpose of this work, we shall consider a fixed time instant corresponding to the systolic peak, which is of crucial clinical interest.

The Figure 2 shows the reconstruction from MRI data of the considered cross-section of the carotid artery; it also displays the spatial locations of the beams inspected in the ECD scan. In particular, during the ECD scan 7 beams are considered, located in a cross-shaped pattern; this unusual pattern is a compromise decided together with clinicians in order to obtain as many observations as possible in the short time dedicated to the acquisition. In the figure each beam is colored according to the value of the mean velocity registered within the beam at the fixed time instant considered, the systolic peak.

In this applied problem there are important conditions at the boundary of the problem domain, i.e., specifically, at the wall of the carotid cross-section represented in Figure 2. The physics of the problem implies in fact that blood-flow velocity is zero at the arterial wall, due to the friction between blood cells and arterial wall; these are the so-called no-slip boundary conditions.

Classical methods for surface estimation, as tensor product of unidimensional splines, thin-plate splines, bidimensional kernel smoothing, bidimensional wavelet-based smoothing and kriging, are naturally defined on tensorized domains and do not efficiently deal with more complex domains, when the shape of the domain is important for the behavior of the phenomenon under study. Moreover, they cannot naturally include information on the value of the surface at the boundary, although it is possible to enforce such boundary conditions for example with binning. Recently, some methods have been proposed where the shape of the domain and the boundary conditions are instead directly specified in the model. For instance, Finite Element Lsplines described in Ramsay (2002) account explic- 
itly for the shape of the domain, efficiently dealing with irregular shaped domains; soap-film smoothing (SOAP), described in Wood et al. (2008), considers both the shape of the domain and some common types of boundary conditions; Spatial Spline Regression (SSR), presented in Sangalli et al. (2013), extends Ramsay (2002) and includes general boundary conditions. The methods in Ramsay (2002), Wood et al. (2008) and Sangalli et al. (2013) are penalized regression methods with a roughness term involving the Laplacian of the field, the Laplacian being a simple form of partial differential operator that provides a measure of the local curvature of the field. Although being able to account for the shape of the domain and to comply with the required boundary conditions, these methods do not provide physiological estimates of the blood flow velocity field. Figure 3 shows for example the velocity field estimated using SSR. The penalization of a measure of the local curvature of the field oversmooths and flattens the field toward a plane in those regions of the domain where no observations are available; the resulting estimated velocity field has thus rhomboidal isolines, which are certainly non-physiological.

On the other hand, we have prior knowledge on the phenomenon under study that could be exploited to derive accurate physiological estimates. There is in fact a vast literature devoted to the study of fluid dynamics and hemodynamics, see for example Formaggia et al. (2009) and references therein. For what serves our purpose, it suffices to know that the theoretical solution of a stationary velocity field in a straight pipe with circular section has a parabolic profile. In our application, during the systolic phase, we hence expect to obtain a velocity field similar in

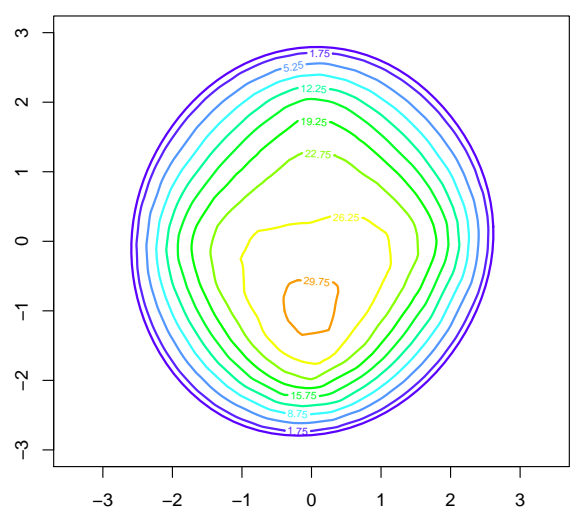

Figure 3: Estimate of the blood-flow velocity field on the carotid section using standard SSR.

shape to a parabolic profile, with smooth isolines resembling circles. Notice that the real blood velocity field is not perfectly parabolic due to the curvature of the artery, the non-stationarity of the blood flow and the imperfect circularity of the artery section. For instance, the curvature of the artery and the non-stationarity in time of the blood flow may induce asymmetries and eccentricities in the blood flow. For this reason, imposing a parametric model that forces the estimate to be a parabolic profile with circular isolines would not be appropriate; such a model would, for instance, completely miss the physiological asymmetries and eccentricities of the flow. Nevertheless, this prior information concerning the shape of the field, which can be conveniently translated into a partial differential operator, could be incorporated in a non-parametric model, along with the desired boundary conditions. Partial Differential Equations (PDEs) are a multidimensional extension of Ordinary Differential Equations (ODEs); their study though requires different and more complex analyt- 
ical and numerical techniques with respect to those used for ODEs; see, e.g., Evans (1998).

In this work, extending Ramsay (2002) and Sangalli et al. (2013), we propose a non-parametric model that includes prior information on the phenomenon under study, coming for instance from the physics, physiology, mechanics or chemistry of the problem, and formalized in terms of a governing PDE. Specifically, Spatial Regression with PDE penalization (SR-PDE) features a roughness term that involves, instead of the simple Laplacian, a more general PDE modeling the phenomenon. The applicability of the proposed method is by no way restricted to the problem here considered; PDEs are in fact commonly used to describe complex phenomena behavior in many fields of engineering and sciences, including bio-sciences, geo-sciences and physical sciences. Applications of particular interest in the environmental sciences concern e.g. the study of the dispersion of pollutant released in water or in air and transported by streams or winds, the study of temperature, pressure and electromagnetic fields, the study of the propagation of earthquakes, tsunamis and other wave phenomena. Important applications in biomedicine include also the study of electrophysiology and mechanics of organs and the study of tumor growth dynamics. It should be noticed that many methods for surface and spatial field estimation, besides the already cited SSR and SOAP, use roughness penalties involving some simple form of PDEs. A classical example is given by thin-plate-splines, while a recent proposal is offered for instance by Guillas and Lai (2010). Our work has also strong connections with the framework introduced by Lindgren et al. (2011), and more generally with the larger literature on Bayesian inverse problem, see, e.g., Stuart (2010). In particular, Lindgren et al. (2011) proposes Bayesian spatial models that link Gaussian fields and Gaussian Markov random fields via a stochastic PDE inducing a Matérn covariance. One important novelty of the proposed SR-PDE models with respect to the methods cited above is that the PDE is here used to model the space variation of the phenomenon, using problem-specific information. Moreover SR-PDE allows for important modeling flexibility in this respect, accounting also for space anisotropy and nonstationarity in a straightforward way, as well as unidirectional smoothing effects. The approach used to solve the surface estimation problem borrows techniques from PDEs control theory, see for example Hinze et al. (2009), Lions (1971) and Quarteroni (2008). However, the viewpoint that we have in this work is quite different from the classical control theory framework, where the main interest is focused on surface estimation starting from integral targets, usually known without error, instead of partial, pointwise and noisy observations. For this reason, different questions have to be addressed in our framework, with respect to those classically considered in control theory. Finally, the smoothing method here proposed could in principle be further extended also to a larger class of penalty terms, including higher-order operators or non-linear operators, provided that the associated differential problems are well posed. However, their numerical approximation may become much more involved.

Likewise in Ramsay (2002) and Sangalli et al. (2013), SR-PDE exploits advanced numerical analysis techniques and, specifically, it makes use of the 
Finite Element Method, which provides a basis for piecewise polynomial surfaces. The resulting estimators have a penalized regression form, they are linear in the observed data values and classical inferential tools can be derived. The proposed method is currently implemented in $\mathrm{R}$, see $\mathrm{R}$ Development Core Team (2012), and in FreeFem++, see Pironneau et al. (2011).

The paper is organized as follows. Section 2 introduces SR-PDE for pointwise observations. Section 3 extends the models to the case of areal data, which is of interest in many applications, including the analysis of ECD measurements here considered. Section 4 describes the Finite Element solution to the estimation problem and derives the inferential properties of the estimators. Section 5 deals with general boundary conditions. In Section 6, SR-PDE is compared to standard SSR and to SOAP in different simulation settings, with data distributed uniformly on the domain or only on some subregions, showing that the inclusion of the prior knowledge on the phenomenon behavior improves significantly the estimates. In Section 7 the application within the MACAREN@MOX project is presented: details on the ECD acquisitions are given and the results obtained with SR-PDE are shown. Section 8 outlines future research directions.

\section{Model for pointwise data}

Consider a bounded and regular domain $\Omega \subset \mathbb{R}^{2}$, whose boundary $\partial \Omega$ is a curve of class $C^{2}$, and $n$ observations $z_{i}$, for $i=1, \ldots, n$, located at points $\mathbf{p}_{i}=\left(x_{i}, y_{i}\right) \in \Omega$. Assume the model

$$
z_{i}=f_{0}\left(\mathbf{p}_{i}\right)+\epsilon_{i}
$$

where $\epsilon_{i}, i=1, \ldots, n$, are independent errors with zero mean and constant variance $\sigma^{2}$, and $f_{0}: \Omega \rightarrow \mathbb{R}$ is the surface or spatial field to be estimated. In our application, $\Omega$ will be the carotid cross-section of interest, the observations $z_{i}$ will represent the blood particles velocities measured by ECD in the longitudinal direction of the artery (i.e., in the orthogonal direction to $\Omega$ ) and the surface $f_{0}$ will represent the longitudinal velocity field on the carotid crosssection.

Assume that problem specific prior information is available, that can be described in terms of a PDE, $L f_{0}=u$, modeling to some extent the phenomenon under study; moreover, prior knowledge could also concern possible conditions that $f_{0}$ has to satisfy at the boundary $\partial \Omega$ of the problem domain. Generalizing the models in Ramsay (2002) and Sangalli et al. (2013), we propose to estimate $f_{0}$ by minimizing the penalized sum-of-square-error functional

$$
J(f)=\sum_{i=1}^{n}\left(f\left(\mathbf{p}_{i}\right)-z_{i}\right)^{2}+\lambda \int_{\Omega}(L f(\mathbf{p})-u(\mathbf{p}))^{2} d \mathbf{p}
$$

with respect to $f \in V$, where $V$ is the space of functions in $L^{2}(\Omega)$ with first and second derivatives in $L^{2}(\Omega)$, that satisfy the required boundary conditions (b.c.). The penalized error functional hence trades off a data fitting criterion, the sum-of-squareerror, and a model fitting criterion, that penalizes departures from a PDE problem-specific description of the phenomenon. Because of the inclusion of a PDE in the definition of the statistical model, the proposed method can be seen as a regularized least square analogous to the Bayesian inverse problems presented, e.g., in Stuart (2010). In particular, the least square term in $J(f)$ corresponds to a log- 
likelihood for Gaussian errors, while the regularizing term effectively translates the prior knowledge on the surface. With respect to Stuart (2010), besides the different model framework and estimation approaches, we also deal with a larger class of operators, including non-stationary anisotropic diffusion, transport and reaction terms.

In particular, we consider here phenomena that are well described in terms of linear second order elliptic operators $L$ and forcing term $u \in L^{2}(\Omega)$ that can be either $u=0$, homogeneous case, or $u \neq 0$, non-homogeneous case. The operator $L$ is a general differential operator that can, for instance, include second, first and zero order differential operators. Consider a symmetric and positive definite matrix $\mathbf{K}=\left\{K_{i j}\right\}_{i, j=1,2} \in \mathbb{R}^{2 \times 2}$, named diffusion tensor, a vector $\mathbf{b}=\left(b_{1}, b_{2}\right) \in \mathbb{R}^{2}$, named transport vector, and a positive scalar $c \in \mathbb{R}^{+}$, named reaction term. Then, the operator can include: second order differential operators as the divergence of the gradient, i.e.,

$$
\begin{aligned}
\operatorname{div}(\mathbf{K} \nabla f)= & \frac{\partial}{\partial x}\left(K_{11} \frac{\partial f}{\partial x}+K_{12} \frac{\partial f}{\partial y}\right)+ \\
& \frac{\partial}{\partial y}\left(K_{21} \frac{\partial f}{\partial x}+K_{22} \frac{\partial f}{\partial y}\right)
\end{aligned}
$$

first order differential operators as the gradient, i.e.,

$$
\mathbf{b} \cdot \nabla f=b_{1} \frac{\partial f}{\partial x}+b_{2} \frac{\partial f}{\partial y},
$$

and also zero order operators, i.e., $c f$. The general form that we consider is

$$
L f=-\operatorname{div}(\mathbf{K} \nabla f)+\mathbf{b} \cdot \nabla f+c f .
$$

The three terms that compose the general second order operator (3) induce an anisotropic and nonstationary smoothing, providing different regularizing effects. The diffusion term $-\operatorname{div}(\mathbf{K} \nabla f)$ induces a smoothing in all the directions; if the diffusion matrix $\mathbf{K}$ is a multiple of the identity the diffusion term has an isotropic smoothing effect, otherwise it implies an anisotropic smoothing with a preferential direction that corresponds to the first eigenvector of the diffusion tensor $\mathbf{K}$. The degree of anisotropy induced by the diffusion tensor $\mathbf{K}$ is controlled by the ratio between its first and second eigenvalue. It is possible to visualize the diffusion term as the quadratic form in $\mathbb{R}^{2}$ induced by the tensor $\mathbf{K}^{-1}$. On the contrary the transport term $\mathbf{b} \cdot \nabla f$ induces a smoothing only in the direction specified by the transport vector $\mathbf{b}$. Finally, the reaction term $c f$ has instead a shrinkage effect, since penalization of the $L^{2}$ norm of $f$ induces a shrinkage of the surface to zero. Setting $\mathbf{K}=\mathbf{I}, \mathbf{b}=\mathbf{0}, c=0$ and $u=0$ we obtain the special case described in Ramsay (2002) and Sangalli et al. (2013), where the penalization of the Laplacian $\Delta f$ induces an isotropic and stationary smoothing.

The parameters of the differential operator $L$ can also be space-varying on $\Omega$; i.e., $\mathbf{K}=\mathbf{K}(x, y)$, $\mathbf{b}=\mathbf{b}(x, y)$ and $c=c(x, y)$. This feature is fundamental to translate the a priori information on the phenomenon. For instance, in the blood flow velocity application, the problem specific prior information can be described via an appropriate anisotropic and non-stationary differential operator that includes: a non-stationary anisotropic diffusion tensor that smooths the observations in the tangential direction of concentric circles (see Figure 4 Center); a non-stationary transport field that smooths the 

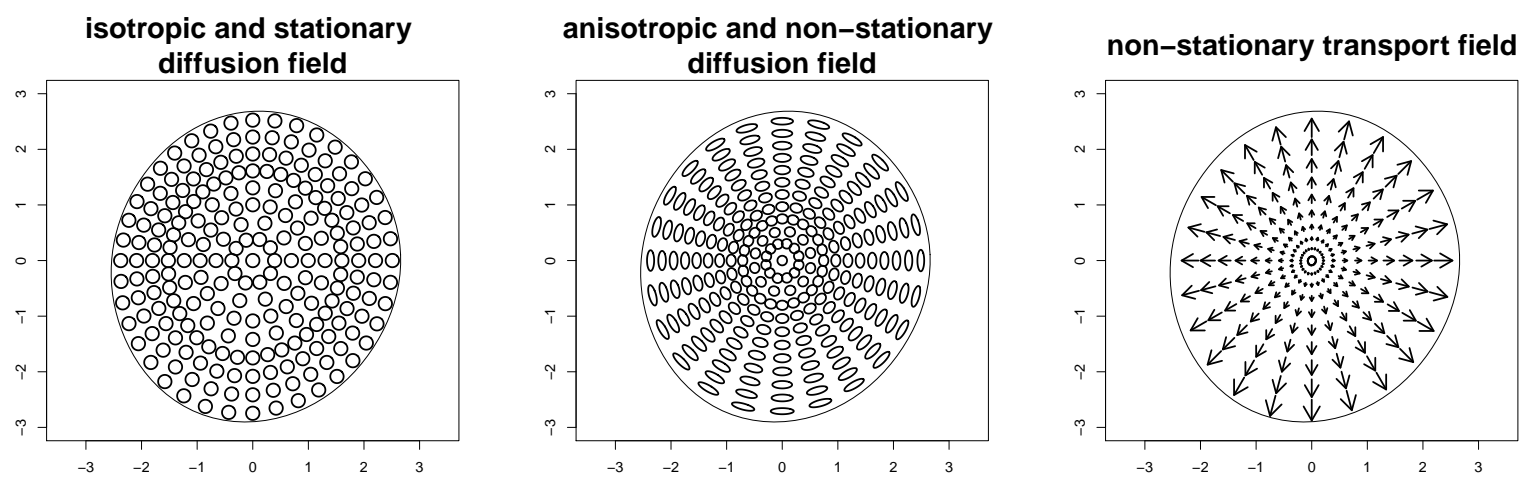

Figure 4: Left: stationary and isotropic diffusion tensor $\mathbf{K}=\mathbf{I}$ used in the blood-flow velocity field application for SSR. Center and Right: non-stationary and anisotropic diffusion tensor field $\mathbf{K}$ and nonstationary transport field $\mathbf{b}$, used in the blood-flow velocity field application for SR-PDE: the displayed tensor field $\mathbf{K}$ smooths the observations in the tangential direction of concentric circles, while the transport field $\mathbf{b}$ smooths the observations in the radial direction, from the center of the carotid section to the arterial wall.

observations in the radial direction, from the center of the section to the boundary (see Figure 4 Right). The reaction term is instead not required in this application. The non-stationary and anisotropic diffusion tensor field $\mathbf{K}$ used in the application and represented in the central panel of Figure 4 can be compared to the stationary and isotropic diffusion tensor field $\mathbf{K}=\mathbf{I}$ used in SSR and represented in the left panel of Figure 4. Notice that the space-varying parameters need to satisfy some regularity conditions to ensure that the solution of the corresponding PDE is regular enough for the estimation problem to be well-posed (see Appendix A for details). The functional $J(f)$ is well defined if $f \in V$ since $V \subset C(\bar{\Omega})$ if $\Omega \subset \mathbb{R}^{2}$ and the misfit of the PDE is square integrable.

We can impose different types of boundary conditions, homogeneous or not, that involve the evaluation of the function and/or its first derivative at the boundary, allowing for a complex modeling of the behavior of the surface at the boundary $\partial \Omega$ of the domain. For ease of notation we consider in the following the simple case of homogeneous Dirichlet b.c., which involve the value of the function at the boundary, clamping it to zero, i.e., $\left.f\right|_{\partial \Omega}=0$. These boundary conditions correspond to the physiological no-slip conditions needed in the ECD application; the blood cells have in fact zero longitudinal velocity near the arterial wall due to friction between the blood particles and the arterial wall. In Appendix C we extend all the results presented in this section to the case of more general non-homogeneous boundary conditions that can also involve first derivatives. In this work the boundary conditions are directly included in the space $V$; in the case of Dirichlet homogeneous b.c., $V$ is the space of functions in $L^{2}(\Omega)$ with first and second derivatives in $L^{2}(\Omega)$ and zero value at the boundary $\partial \Omega$.

To lighten the notation, surface integrals will be written without the integration variable $\mathbf{p}$; unless differently specified, the integrals are computed with respect of the Lebesgue measure, i.e., $\int_{D} q=$ $\int_{D} q(\mathbf{p}) d \mathbf{p}$, for any $D \subseteq \mathbb{R}^{2}$ and integrable function $q$. 
All the results presented can also be extended to include space-varying covariate information, following the semi-parametric approach described in Sangalli et al. (2013).

\subsection{Solution to the estimation problem}

The estimation problem can be formulated as follows.

Problem 1. Find $\hat{f} \in V$ such that

$$
\hat{f}=\underset{f \in V}{\operatorname{argmin}} J(f) .
$$

Proposition 1. Under suitable regularity conditions for $L$, the solution of Problem 1 exists and is unique.

The required regularity conditions, the proof of Proposition 1 and the solution of the estimation problem are detailed in Appendix A. The key idea consists in exploiting the linearity of the PDE penalized in the roughness term in order to prove the convexity of the functional $J(f)$. The minimum is then obtained by differentiating the functional $J(f)$ with respect to $f$. The differentiation of the functional $J(f)$ provides an explicit expression for the surface estimate $\hat{f}$ as the solution of a linear fourth order problem. A convenient way to solve this fourth order problem is to split it into the following coupled system of second order PDEs:

$$
\begin{aligned}
& \begin{cases}L \hat{f}=u+\hat{g} & \text { in } \Omega \\
\hat{f}=0 & \text { on } \partial \Omega\end{cases} \\
& \begin{cases}L^{*} \hat{g}=-\frac{1}{\lambda} \sum_{i=1}^{n}\left(\hat{f}-z_{i}\right) \delta_{\mathbf{p}_{i}} & \text { in } \Omega \\
\hat{g}=0 & \text { on } \partial \Omega\end{cases}
\end{aligned}
$$

where $\hat{g}$ represents the misfit of the penalized PDE, i.e., $\hat{g}=L \hat{f}-u, L^{*}$ is the adjoint operator of $L$, i.e., is such that $\int_{\Omega} L \varphi \psi=\int_{\Omega} \varphi L^{*} \psi$ for all $\varphi, \psi \in V$, and is defined as

$$
L^{*} \hat{g}=-\operatorname{div}(\mathbf{K} \nabla \hat{g})-\mathbf{b} \cdot \nabla \hat{g}+(c-\operatorname{div}(\mathbf{b})) \hat{g}
$$

The coupled system of PDEs (4) is then solved by means of the Finite Element method, which is a standard technique used to solve PDEs (see, e.g., Quarteroni (2008)), in order to obtain a piecewise polynomial discretization of the surface estimate.

\section{Model for areal data}

We here extend the surface smoothing method presented in the previous Section to the case of areal data, a setting common in many applications, including the one driving our study.

Let $D_{i} \subset \Omega$, for $i=1, \ldots, N$, be some subdomains where we have observations and $z_{i j}$, for $j=1, \ldots, n_{i}$, be the observations located at point $\mathbf{p}_{i j} \in D_{i}$. For the observations $z_{i j}$, we consider the pointwise model (1), i.e.,

$$
z_{i j}=f_{0}\left(\mathbf{p}_{i j}\right)+\epsilon_{i j}
$$

where $\epsilon_{i j}$, for $i=1, \ldots, N$ and $j=1, \ldots, n_{i}$, are independent errors with zero mean and constant variance $\sigma^{2}$.

In the blood flow velocity application, the location points $\mathbf{p}_{i j}$ are unknown, the only available information being that $\mathbf{p}_{i j} \in D_{i}$, where $D_{i}$ is the $i$-th ECD acquisition beam. We may assume that the location points $\mathbf{p}_{i j}$ are distributed over the subdomains according to a global uniform distribution over $\Omega$ and that the subdomains are not overlapping. For each beam $D_{i}$, the ECD signal (Figure 1) provides, at a 
fixed time, a histogram of the measured blood particle velocities. We summarize the information carried by the histogram by its mean value. Specifically, let $\bar{z}_{i}$ be the mean value of the observations on the subdomain $D_{i}$, for $i=1, \ldots, N$. From (6), we can derive the following model for this variable:

$$
\bar{z}_{i}=\frac{1}{n_{i}} \sum_{j=1}^{n_{i}} f_{0}\left(\mathbf{p}_{i j}\right)+\frac{1}{n_{i}} \sum_{j=1}^{n_{i}} \epsilon_{i j}
$$

where $\sum_{j=1}^{n_{i}} \epsilon_{i j} / n_{i}, i=1, \ldots, N$, are errors with zero mean and variance $\sigma^{2} / n_{i}$.

The quantity $\sum_{j=1}^{n_{i}} f_{0}\left(\mathbf{p}_{i j}\right) / n_{i}$ is the Monte Carlo approximation of $\mathbb{E}\left[f_{0}(P) \mid P \in D_{i}\right]$ and the latter is in turn equal to the spatial average of the surface on the subdomain $D_{i}$, under the assumption of uniformly distributed observation points, i.e.,

$\frac{1}{n_{i}} \sum_{j=1}^{n_{i}} f_{0}\left(\mathbf{p}_{i j}\right) \approx \mathbb{E}\left[f_{0}(P) \mid P \in D_{i}\right]=\frac{1}{\left|D_{i}\right|} \int_{D_{i}} f_{0}$.

We may thus consider the following model:

$$
\bar{z}_{i}=\frac{1}{\left|D_{i}\right|} \int_{D_{i}} f_{0}+\eta_{i}
$$

where the error terms $\eta_{i}$ have zero mean and variances $\bar{\sigma}_{i}^{2}$ inversely proportional to the dimension of the beams $D_{i}$; this assumption on the variances is coherent with the assumption on location points being distributed on the subdomains according to a uniform distribution (so that in fact the average number of observations on each subdomain is proportional to the dimension of the subdomain). If the subdomains have the same dimension, as it is in fact the case in our application, this simplifies to variances all equal to $\bar{\sigma}^{2}$.

In order to estimate the surface we hence propose to minimize the penalized sum-of-square-error functional

$$
\bar{J}(f)=\sum_{i=1}^{N} \frac{1}{\left|D_{i}\right|}\left(\int_{D_{i}}\left(f-\bar{z}_{i}\right)\right)^{2}+\lambda \int_{\Omega}(L f-u)^{2}
$$

with respect to $f \in V$. The first term is now a weighted least-square-error functional for areal data on the subdomains $D_{i}$, where the weights are in fact equal to the inverse of the variances $\bar{\sigma}_{i}^{2}$, being $\bar{\sigma}_{i}^{2} \propto 1 /\left|D_{i}\right|$. Notice that the functional (8) mixes two different kinds of information: the data provide information only on the areal means of the surface $f$ over the subdomains, while the roughness penalty translates the prior knowledge directly on the shape of $f$.

\subsection{Solution to the estimation problem}

The estimation problem can be formulated as follows.

Problem 2. Find $\hat{f} \in V$ such that

$$
\hat{f}=\underset{f \in V}{\operatorname{argmin}} \bar{J}(f) .
$$

\section{Proposition 2. Under suitable regularity conditions} for L, the solution of Problem 2 exists and is unique.

Details on the regularity conditions and on the proof are provided in Appendix A. The proof follows the same strategy used in the proof of Proposition 1. Moreover, using the same arguments as for the pointwise case it can be shown that the surface estimator $\hat{f}$ can be obtained by solving a fourth order problem and that the latter problem can be conveniently split into the following coupled system of second order 
problems:

$$
\begin{aligned}
& \begin{cases}L \hat{f}=u+\hat{g} & \text { in } \Omega \\
\hat{f}=0 & \text { on } \partial \Omega\end{cases} \\
& \begin{cases}L^{*} \hat{g}=-\frac{1}{\lambda} \sum_{i=1}^{N} \frac{1}{\left|D_{i}\right|} \mathbb{I}_{D_{i}} \int_{D_{i}}\left(\hat{f}-\bar{z}_{i}\right) & \text { in } \Omega \\
\hat{g}=0 & \text { on } \partial \Omega\end{cases}
\end{aligned}
$$

where $\hat{g}$ represents the misfit of the PDE penalized, i.e., $\hat{g}=L \hat{f}-u$, and $L^{*}$ is the adjoint operator of $L$, defined in (5). As in the pointwise case the coupled system of PDEs (9) is then discretized by means of the Finite Element method.

Remark 1. All the results presented in this section can be extended to the case of location points distributed on the subdomains according to a general known global distribution $\mu$ over $\Omega, P \sim$ $\mu$. The quantity $\sum_{j=1}^{n_{i}} f_{0}\left(\mathbf{p}_{i j}\right) / n_{i}$ is in fact, also in this case, the Monte Carlo approximation of $\mathbb{E}\left[f_{0}(P) \mid P \in D_{i}\right]$ :

$$
\begin{aligned}
\frac{1}{n_{i}} \sum_{j=1}^{n_{i}} f_{0}\left(\mathbf{p}_{i j}\right) & \approx \mathbb{E}\left[f_{0}(P) \mid P \in D_{i}\right] \\
& =\frac{1}{\mu\left(D_{i}\right)} \int_{D_{i}} f_{0}(\mathbf{p}) \mu(d \mathbf{p}) .
\end{aligned}
$$

Therefore the model for the areal mean on the subdomains becomes:

$$
\bar{z}_{i}=\frac{1}{\mu\left(D_{i}\right)} \int_{D_{i}} f_{0}(\mathbf{p}) \mu(d \mathbf{p})+\eta_{i} .
$$

Under the assumption of non overlapping subdomains, the errors $\eta_{i}$ have zero mean and variances inversely proportional to $\mu\left(D_{i}\right)$, which is the probability of sampling a point in the subdomain $D_{i}$. The surface estimator $\hat{f}$ can be obtained minimizing the weighted least square functional

$$
\begin{aligned}
\bar{J}_{\mu}(f)= & \sum_{i=1}^{N} \frac{1}{\mu\left(D_{i}\right)}\left(\int_{D_{i}}\left(f(\mathbf{p})-\bar{z}_{i}\right) \mu(d \mathbf{p})\right)^{2}+ \\
& \lambda \int_{\Omega}(L f-u)^{2}
\end{aligned}
$$

with respect to $f \in V$. The weights in the least square term are proportional to the inverse of $\operatorname{Var}\left(\bar{z}_{i}\right)$, being $\operatorname{Var}\left(\bar{z}_{i}\right) \propto 1 / \mu\left(D_{i}\right)$.

\section{Finite Element solution to the esti- mation problem}

The surface estimation problems in the pointwise and areal data frameworks presented respectively in Sections 2 and 3 are infinite dimensional problems and cannot be solved analytically. A standard approach to handle these problems is to reformulate them in a proper finite dimensional subspace that approximates the original infinite dimensional one. This approach is used in many fields of statistics and applied mathematics, e.g., non-parametric statistics, functional and spatial data analysis and analysis of differential equations. In particular the standard method used in numerical analysis to solve PDEs is the Finite Element method (see, e.g., Quarteroni (2008)) which provides a basis for continuous piecewise polynomial surfaces over a triangulation of the domain of interest. The discretization of a surface by means of Finite Elements is similar to the discretization of a curve by means of univariate splines, the latter providing a basis for piecewise polynomial curves.

Let $\mathcal{T}_{h}$ be a triangulation of the domain, where $h$ denotes the characteristic mesh size, defined as the maximum length of the triangle edges in the trian- 


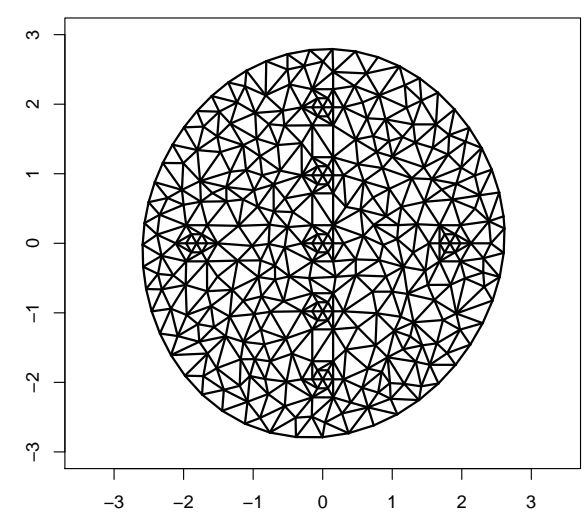

Figure 5: Triangulation of the carotid cross-section used in the blood-flow velocity field application.

gulation. Figure 5 shows the triangulation considered in the velocity field application. We consider the space $V_{h}^{r}$ of piecewise polynomial functions of order $r \geq 1$ over the triangulation. Denoting with $\mathbb{P}^{r}(\boldsymbol{\tau})$ the space of polynomial functions of order $r$ over the triangle $\tau$, the space $V_{h}^{r}$ is defined as

$$
V_{h}^{r}=\left\{v \in C^{0}(\bar{\Omega}):\left.v\right|_{\boldsymbol{\tau}} \in \mathbb{P}^{r}(\boldsymbol{\tau}) \forall \boldsymbol{\tau} \in \mathcal{T}_{h}\right\}
$$

The space $V_{h}^{r}$ is a finite dimensional subspace of $H^{1}(\Omega)$, which is the space of functions in $L^{2}(\Omega)$ with first derivatives in $L^{2}(\Omega)$. The latter space is characterized by less regularity than the space $V$, which has both first and second derivatives in $L^{2}(\Omega)$. It is thus necessary to appropriately reformulate the estimation problems (4) and (9) in order to involve only first order derivatives, as it will be described in Section 4.1 and 4.2.

Let $N_{h}=\operatorname{dim}\left(V_{h}^{r}\right)$ and denote by $\psi_{1}, \ldots, \psi_{N_{h}}$ the Finite Element basis functions such that $V_{h}^{r}=$ $\operatorname{span}\left\{\psi_{1}, \ldots, \psi_{N_{h}}\right\}$. The basis $\psi_{1}, \ldots, \psi_{N_{h}}$ are associated to the nodes $\boldsymbol{\xi}_{1}, \ldots, \boldsymbol{\xi}_{N_{h}}$, that correspond

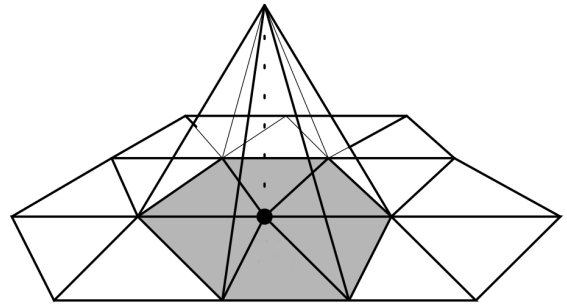

Figure 6: Illustration of a linear Finite Element basis function on a triangulation.

to the vertices of the triangulation $\mathcal{T}_{h}$ if the basis is piecewise linear, and are instead a superset of the vertices when the degree of the polynomial basis is higher than one. Notice that the nodes of the mesh and the mesh itself can be defined independently of the location points $\mathbf{p}_{1}, \cdots, \mathbf{p}_{n}$. Figure 6 shows for example a linear Finite Element basis function on a regular triangulation. The basis functions are local basis, with compact support, that can be seen as multidimensional extensions of univariate splines. Moreover, the basis functions $\psi_{1}, \ldots, \psi_{N_{h}}$ are $\mathrm{La}$ grangian, meaning that $\psi_{k}\left(\xi_{l}\right)=\delta_{k l}$, where $\delta_{i j}=1$ if $i=j$ and $\delta_{i j}=0$ otherwise. Hence a surface $f \in V_{h}^{r}$ is uniquely determined by its values at the nodes:

$$
f(x, y)=\sum_{k=1}^{N_{h}} f\left(\xi_{k}\right) \psi_{k}(x, y)=\boldsymbol{\psi}(x, y)^{T} \mathbf{f}
$$

where

$$
\mathbf{f}=\left(f\left(\boldsymbol{\xi}_{1}\right), \ldots, f\left(\boldsymbol{\xi}_{N_{h}}\right)\right)^{T}
$$

and

$$
\boldsymbol{\psi}=\left(\psi_{1}, \ldots, \psi_{N_{h}}\right)^{T}
$$

In the following we consider only homogeneous Dirichlet b.c., for which the value of the function at the boundary is fixed to 0 . In this case we consider the Finite Element space 
$V_{h, 0}^{r}=\left\{v \in C^{0}(\bar{\Omega}):\left.v\right|_{\partial \Omega}=0\right.$ and $\left.v\right|_{\boldsymbol{\tau}} \in \mathbb{P}^{r}(\boldsymbol{\tau})$ $\left.\forall \boldsymbol{\tau} \in \mathcal{T}_{h}\right\}$, of dimension $N_{h, 0}$, which only necessitates of the basis functions associated to the internal nodes of the triangulation, whilst the basis functions associated to the boundary nodes can be discarded. In Appendix $\mathrm{C}$ we extend the results presented in this section to the case of more general boundary conditions.

\subsection{Pointwise estimator}

The Finite Element surface estimator $\hat{f}_{h}$ is obtained recasting the estimation problem in the finite dimensional space $V_{h, 0}^{r}$, thus obtaining a discretized version of the coupled system of PDEs (4). As previously highlighted, the estimation problem (4) is defined in $V$, which is a space with both first and second order derivatives in $L^{2}(\Omega)$, whilst the Finite Element space $V_{h, 0}^{r}$ provides a finite dimensional subspace of $H^{1}(\Omega)$. For this reason we need to reformulate the estimation problem in a form that can be properly discretized using the Finite Element space. This appropriate reformulation of the problem, called variational formulation, involves only first order derivatives and is obtained integrating the differential equations against a test function and integrating by parts the second order terms.

By introducing the test function $\psi \in V_{h, 0}^{r}$ we can define the bilinear form $a(\cdot, \cdot)$, associated to the operator $L$, as

$a\left(\hat{f}_{h}, \psi_{h}\right)=\int_{\Omega}\left(\mathbf{K} \nabla \hat{f}_{h} \cdot \nabla \psi_{h}+\mathbf{b} \cdot \nabla \hat{f}_{h} \psi_{h}+c \hat{f}_{h} \psi_{h}\right)$.

The discrete surface estimators $\hat{f}_{h}, \hat{g}_{h} \in V_{h, 0}^{r}$ are thus obtained solving

$$
\left\{\begin{array}{l}
a\left(\hat{f}_{h}, \psi_{h}\right)-\int_{\Omega} \hat{g}_{h} \psi_{h}=\int_{\Omega} u \psi_{h} \\
\lambda a\left(\varphi_{h}, \hat{g}_{h}\right)+\sum_{i=1}^{n} \hat{f}_{h}\left(\mathbf{p}_{i}\right) \varphi_{h}\left(\mathbf{p}_{i}\right)=\sum_{i=1}^{n} z_{i} \varphi_{h}\left(\mathbf{p}_{i}\right)
\end{array}\right.
$$

for all $\psi_{h}, \varphi_{h} \in V_{h, 0}^{r}$. Exploiting now the Finite Element representation (11) for the discrete estimators $\hat{f}_{h}, \hat{g}_{h} \in V_{h, 0}^{r}$, we are allowed to write the estimation problem as a linear system. Define $\boldsymbol{\psi}_{x}=\left(\partial \psi_{1} / \partial x, \ldots, \partial \psi_{N_{h, 0}} / \partial x\right)^{T}$ and $\boldsymbol{\psi}_{y}=$ $\left(\partial \psi_{1} / \partial y, \ldots, \partial \psi_{N_{h, 0}} / \partial y\right)^{T}$ and the matrices

$$
\begin{aligned}
\mathbf{R}(c) & =\int_{\Omega} c \boldsymbol{\psi} \boldsymbol{\psi}^{T} \\
\mathbf{R}_{x}(\mathbf{b}) & =\int_{\Omega} b_{1} \boldsymbol{\psi} \boldsymbol{\psi}_{x}^{T}, \\
\mathbf{R}_{y}(\mathbf{b}) & =\int_{\Omega} b_{2} \boldsymbol{\psi} \boldsymbol{\psi}_{y}^{T}, \\
\mathbf{R}_{x x}(\mathbf{K}) & =\int_{\Omega} K_{11} \boldsymbol{\psi}_{x} \boldsymbol{\psi}_{x}^{T}, \\
\mathbf{R}_{y y}(\mathbf{K}) & =\int_{\Omega} K_{22} \boldsymbol{\psi}_{y} \boldsymbol{\psi}_{y}^{T}, \\
\mathbf{R}_{x y}(\mathbf{K}) & =\int_{\Omega} K_{12}\left(\boldsymbol{\psi}_{x} \boldsymbol{\psi}_{y}^{T}+\boldsymbol{\psi}_{y} \boldsymbol{\psi}_{x}^{T}\right)
\end{aligned}
$$

Using this notation, the Finite Element matrix associated to the bilinear form $a(\cdot, \cdot)$ in (12) is given by

$$
\begin{aligned}
\mathbf{A}(\mathbf{K}, \mathbf{b}, c) & =\mathbf{R}_{x x}(\mathbf{K})+\mathbf{R}_{x y}(\mathbf{K})+\mathbf{R}_{y y}(\mathbf{K}) \\
& +\mathbf{R}_{x}(\mathbf{b})+\mathbf{R}_{y}(\mathbf{b})+\mathbf{R}(c) .
\end{aligned}
$$

Moreover, define the vectors $\mathbf{z}=\left(z_{1}, \ldots, z_{n}\right)^{T}$, $\mathbf{u}=\int_{\Omega} u \psi$ and the matrices 
and

$$
\boldsymbol{\Psi}=\left[\begin{array}{c}
\boldsymbol{\psi}^{T}\left(\mathbf{p}_{1}\right) \\
\vdots \\
\boldsymbol{\psi}^{T}\left(\mathbf{p}_{n}\right)
\end{array}\right]
$$

where $\boldsymbol{\Psi}$ is the matrix of basis evaluations at the $n$ data locations $\mathbf{p}_{1}, \cdots, \mathbf{p}_{n}$.

The Finite Element solution $\hat{f}_{h}$ of the discrete counterpart (13) of the estimation Problem 1 is thus given by $\hat{f}_{h}=\boldsymbol{\psi}^{T} \hat{\mathbf{f}}$ where $\hat{\mathbf{f}}$ is the solution of the linear system

$$
\left[\begin{array}{cc}
\boldsymbol{\Psi}^{T} \boldsymbol{\Psi} & \lambda \mathbf{A}^{T} \\
\mathbf{A} & -\mathbf{R}
\end{array}\right]\left[\begin{array}{l}
\hat{\mathbf{f}} \\
\hat{\mathbf{g}}
\end{array}\right]=\left[\begin{array}{c}
\boldsymbol{\Psi}^{T} \mathbf{z} \\
\mathbf{u}
\end{array}\right]
$$

\subsubsection{Properties of the estimator}

The estimator $\hat{f}_{h}$ is a linear function of the observed data values. The fitted values $\hat{\mathbf{z}}=\boldsymbol{\Psi} \hat{\mathbf{f}}$ can be represented as

$$
\hat{\mathbf{z}}=\mathbf{S z}+\mathbf{r}
$$

where the smoothing matrix $\mathbf{S} \in \mathbb{R}^{n \times n}$ and the vector $\mathbf{r} \in \mathbb{R}^{n}$ are obtained as

$$
\begin{aligned}
& \mathbf{S}=\boldsymbol{\Psi}\left(\boldsymbol{\Psi}^{T} \boldsymbol{\Psi}+\lambda \mathbf{P}\right)^{-1} \boldsymbol{\Psi}^{T} \\
& \mathbf{r}=\boldsymbol{\Psi}\left(\boldsymbol{\Psi}^{T} \boldsymbol{\Psi}+\lambda \mathbf{P}\right)^{-1} \lambda \mathbf{P} \mathbf{A}^{-1} \mathbf{u}
\end{aligned}
$$

with $\mathbf{P}$ denoting the penalty matrix

$$
\mathbf{P}=\mathbf{P}(\mathbf{K}, \mathbf{b}, c)=\mathbf{A}^{T}(\mathbf{R})^{-1} \mathbf{A} .
$$

The smoothing matrix $\mathbf{S}$ has the typical form obtained in a penalized regression problem. In particular, the positive definite penalty matrix $\mathbf{P}$ represents the discretization of the penalty term in (2). Notice that, thanks to the variational formulation of the es- timation problem, this penalty matrix does not involve the computation of second order derivatives. Appendix B shows that, in the Finite Element space used to discretize the problem, $\mathbf{P}$ is in fact equivalent to the penalty matrix $\tilde{\mathbf{P}}$ that would be obtained as direct discretization of the penalty term in (2), and involving the computation of second order derivatives. Finally, the vector $\mathbf{r}$ is equal to zero when the penalized PDE is homogeneous $(u=0)$; notice that when no specific information on the forcing term is available, it is indeed preferable to consider homogeneous PDEs.

Thanks to the linearity of the estimator $\hat{\mathbf{z}}$ in the observations, we can easily derive the properties of the estimator and obtain classical inferential tools as pointwise confidence bands and prediction intervals (see also Sangalli et al. (2013)). Let $\mathbf{z}_{0}=$ $\left(f_{0}\left(\mathbf{p}_{1}\right), \ldots, f_{0}\left(\mathbf{p}_{n}\right)\right)^{T}$ be the column vector of evaluations of the true function $f_{0}$ at the $n$ data locations. Recalling that in our model definition $\mathbb{E}[\mathbf{z}]=\mathbf{z}_{0}$ and $\operatorname{Cov}(\mathbf{z})=\sigma^{2} \mathbf{I}$, we can compute the expected value and the variance of the estimator $\hat{\mathbf{z}}$ :

$$
\mathbb{E}[\hat{\mathbf{z}}]=\mathbf{S f}_{0}+\mathbf{b} \quad \text { and } \quad \mathbb{C o v}(\hat{\mathbf{z}})=\sigma^{2} \mathbf{S S}^{T} .
$$

Since we are dealing with linear estimators, we can use $\operatorname{tr}(\mathbf{S})$ as a measure of the equivalent degrees of freedom for linear estimators (see, e.g., Buja et al. (1989) and Hastie and Tibshirani (1990)). Hence we can estimate $\sigma^{2}$ as

$$
\hat{\sigma}^{2}=\frac{1}{n-\operatorname{tr}(\mathbf{S})}(\hat{\mathbf{z}}-\mathbf{z})^{T}(\hat{\mathbf{z}}-\mathbf{z}) .
$$

The smoothing parameter $\lambda$ may be selected via Gen- 
eralized Cross-Validation minimizing the index

$$
G C V(\lambda)=\frac{1}{n(1-\operatorname{tr}(\mathbf{S}) / n)^{2}}(\hat{\mathbf{z}}-\mathbf{z})^{T}(\hat{\mathbf{z}}-\mathbf{z}) .
$$

\subsection{Areal estimator}

Analogously to the case of pointwise observations, also with areal observations we can introduce an equivalent variational formulation of the estimation problem. Specifically, the variational problem associated to (9) can be discretized as

$$
\left\{\begin{array}{c}
a\left(\hat{f}_{h}, \psi_{h}\right)-\int_{\Omega} \hat{g}_{h} \psi_{h}=\int_{\Omega} u \psi_{h} \\
\lambda a\left(\varphi_{h}, \hat{g}_{h}\right)+\sum_{i=1}^{N} \frac{1}{\left|D_{i}\right|} \int_{D_{i}} \hat{f}_{h} \int_{D_{i}} \varphi_{h} \\
=\sum_{i=1}^{N} \bar{z}_{i} \int_{D_{i}} \varphi_{h}
\end{array}\right.
$$

for all $\psi_{h}, \varphi_{h} \in V_{h, 0}^{r}$, where $\hat{f}_{h}, \hat{g}_{h} \in V_{h, 0}^{r}$ and $a(\cdot, \cdot)$ is the bilinear form defined in (12).

Let $\overline{\mathbf{z}}=\left(\bar{z}_{1}, \ldots, \bar{z}_{N}\right)^{T}$ be the vector of mean values on subdomains $D_{1}, \ldots, D_{N}$, and

$$
\bar{\Psi}=\left[\begin{array}{c}
\frac{1}{\left|D_{1}\right|} \int_{D_{1}} \boldsymbol{\psi}^{T} \\
\vdots \\
\frac{1}{\left|D_{N}\right|} \int_{D_{N}} \boldsymbol{\psi}^{T}
\end{array}\right]
$$

be the matrix of spatial means of the basis functions on the subdomains; moreover, introduce the weight matrix $\mathbf{W}=\operatorname{diag}\left(\left|D_{1}\right|, \ldots,\left|D_{N}\right|\right)\left(\right.$ recall that $\bar{\sigma}_{i}^{2} \propto$ $\left.1 /\left|D_{i}\right|\right)$.

The Finite Element solution $\hat{f}_{h}$ of the discrete counterpart of the estimation Problem 2 is thus given by $\hat{f}_{h}=\boldsymbol{\psi}^{T} \hat{\mathbf{f}}$ where $\hat{\mathbf{f}}$ is the solution of the linear system

$$
\left[\begin{array}{cc}
\overline{\mathbf{\Psi}}^{T} \mathbf{W} \overline{\mathbf{\Psi}} & \lambda \mathbf{A}^{T} \\
\mathbf{A} & -\mathbf{R}
\end{array}\right]\left[\begin{array}{l}
\hat{\mathbf{f}} \\
\hat{\mathbf{g}}
\end{array}\right]=\left[\begin{array}{c}
\overline{\mathbf{\Psi}}^{T} \mathbf{W} \overline{\mathbf{z}} \\
\mathbf{u}
\end{array}\right]
$$

Notice that even though the method provides a pointwise surface estimator $\hat{f}_{h}$, in the areal data framework we are in fact interested in the estimator of the spatial mean of the surface on a subdomain $D$ :

$$
\hat{\bar{f}}(D)=\frac{1}{|D|} \int_{D} \hat{f}
$$

The Finite Element counterpart of this estimator is defined as

$$
\hat{\bar{f}}_{h}(D)=\frac{1}{|D|} \int_{D} \hat{f}_{h}=\overline{\boldsymbol{\psi}}_{D}^{T} \hat{\mathbf{f}}
$$

where $\bar{\psi}_{D}=\left(1 /|D| \int_{D} \psi_{1}, \ldots, 1 /|D| \int_{D} \psi_{N_{h, 0}}\right)^{T}$.

\subsubsection{Properties of the estimator}

The discrete surface estimator $\hat{f}_{h}$ and the estimator of the spatial average on the subdomains $\hat{\bar{f}}_{h}$ are linear in the observed data values $\overline{\mathbf{z}}$. The fitted values of the spatial average on the subdmains $D_{1}, \ldots, D_{N}$ are defined as $\hat{\overline{\mathbf{z}}}=\overline{\mathbf{\Psi}} \hat{\mathbf{f}}=\left(\hat{\bar{f}}_{h}\left(D_{1}\right), \ldots, \hat{\bar{f}}_{h}\left(D_{N}\right)\right)^{T}$. They can be represented as

$$
\hat{\overline{\mathbf{z}}}=\overline{\mathbf{S}} \overline{\mathbf{z}}+\overline{\mathbf{r}}
$$

where $\overline{\mathbf{S}} \in \mathbb{R}^{N \times N}$ and $\overline{\mathbf{r}} \in \mathbb{R}^{N}$ are defined as

$$
\begin{aligned}
& \overline{\mathbf{S}}=\overline{\mathbf{\Psi}}\left(\overline{\mathbf{\Psi}}^{T} \mathbf{W} \overline{\mathbf{\Psi}}+\lambda \mathbf{P}\right)^{-1} \overline{\mathbf{\Psi}}^{T} \mathbf{W} \\
& \mathbf{b}=\overline{\mathbf{\Psi}}\left(\overline{\mathbf{\Psi}}^{T} \mathbf{W} \overline{\mathbf{\Psi}}+\lambda \mathbf{P}\right)^{-1} \lambda \mathbf{P A}^{-1} \mathbf{u}
\end{aligned}
$$

From the definition of model (7) and the linearity of the estimator we can derive the mean of the estimator

$$
\mathbb{E}[\hat{\mathbf{z}}]=\overline{\mathbf{S}} \overline{\mathbf{z}}_{0}+\overline{\mathbf{b}}
$$

where $\left[\overline{\mathbf{z}}_{0}\right]_{i}=1 /\left|D_{i}\right| \int_{D_{i}} f_{0}$, and its covariance

$$
\operatorname{Cov}(\hat{\overline{\mathbf{z}}})=\overline{\mathbf{S}} \operatorname{diag}\left(\bar{\sigma}_{1}^{2}, \ldots, \bar{\sigma}_{N}^{2}\right) \overline{\mathbf{S}}^{T}
$$


It should be noticed that in the areal data framework the expected value (17) and the variance (18) refer to the estimator of the spatial mean on a subdomain. In fact, even though we can obtain a pointwise estimator for the surface $\hat{f}_{h}$, we cannot provide an accurate uncertainty quantification for this estimate, because model (7) provides information only on the areal errors $\eta_{i}$. In particular, in the considered areal framework, the variance

$$
\operatorname{Cov}(\hat{\mathbf{f}})=\boldsymbol{\Psi} \overline{\mathbf{\Psi}}^{-1} \overline{\mathbf{S}} \operatorname{diag}\left(\bar{\sigma}_{1}^{2}, \ldots, \bar{\sigma}_{N}^{2}\right) \overline{\mathbf{S}}^{T} \overline{\mathbf{\Psi}}^{-T} \mathbf{\Psi}^{T}
$$

would underestimate the real variance of $\hat{\mathbf{f}}$.

\section{General boundary conditions}

All the results presented in Sections 2, 3 and 4 can be extended to the case of general homogeneous and non-homogeneous boundary conditions involving the value of the surface or of its first derivatives at the boundary $\partial \Omega$, allowing for a complex modeling of the phenomenon behavior at the boundary of the domain. The three classic boundary conditions for second order PDEs are Dirichlet, Neumann and Robin conditions. The Dirichlet condition controls the value of the function at the boundary, i.e., $\left.f\right|_{\partial \Omega}=\gamma_{D}$, the Neumann condition concerns the value of the normal derivative of the function at the boundary, i.e., $\left.\mathbf{K} \nabla f \cdot \boldsymbol{\nu}\right|_{\partial \Omega}=\gamma_{N}$, where $\boldsymbol{\nu}$ is the outward unit normal vector to $\partial \Omega$, while the Robin condition involves the value of a linear combination of first derivative and the value of the function at the boundary, i.e., $\mathbf{K} \nabla f \cdot \boldsymbol{\nu}+\left.\chi f\right|_{\partial \Omega}=\gamma_{R}$. We can also impose different boundary conditions on different boundary portions forming a partition of $\partial \Omega$. All the admissible boundary conditions can be summa- rized as

$$
\begin{cases}f=\gamma_{D} & \text { on } \Gamma_{D} \\ \mathbf{K} \nabla f \cdot \boldsymbol{\nu}=\gamma_{N} & \text { on } \Gamma_{N} \\ \mathbf{K} \nabla f \cdot \boldsymbol{\nu}+\chi f=\gamma_{R} & \text { on } \Gamma_{R}\end{cases}
$$

where $\gamma_{D}, \gamma_{N}$ and $\gamma_{R}$ have to satisfy some regularity conditions in order to obtain a well defined functional $J(f)$ (see, e.g., Evans (1998)).

Under (19), the solution of the estimation problem and of its discrete counterpart also involve boundary terms. Appendix $\mathrm{C}$ gives all the details for this general case.

\section{Simulation studies}

In this section we study the performances of SRPDE, comparing it to standard SSR and to SOAP in simple simulation studies. We focus on simulation studies that mimic our application setting. The domain $\Omega$ is quasi circular; the true surface $f_{0}$, represented in Figure 7, is obtained as a deformation of a parabolic profile using landmark registration and is equal to zero at the boundary of the domain. Likewise for our application, we assume to have a priori information about the shape of the field, that is known to have a quasi parabolic profile, with almost circular isolines, and to be zero at the boundary.

Since SOAP is not currently devised to deal with areal data, we here consider pointwise observations, with location points sampled on the whole or only on subregions of the domain (the supplementary material available online presents simulation studies comparing SR-PDE to standard SSR in the areal data framework). Specifically, we here consider three cases:

A. $n=100$ observation points $\mathbf{p}_{1}, \ldots, \mathbf{p}_{n}$ sampled 

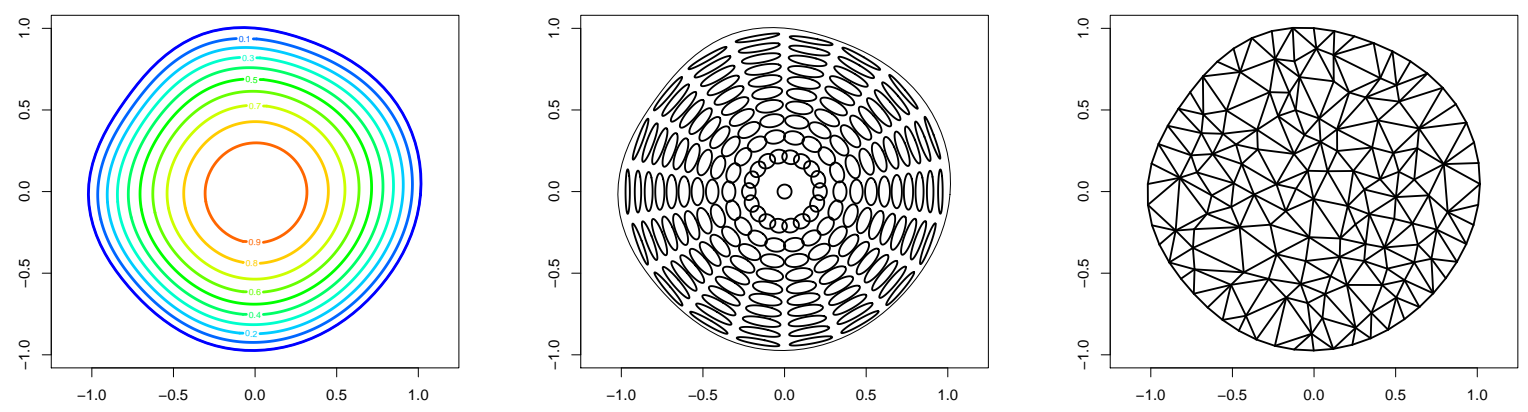

Figure 7: Left: true surface $f_{0}$, with almost circular isolines and zero value at the boundary of the domain, used for the simulation studies; the image displays the isolines $(0,0.1, \ldots, 0.9,1)$. Center: diffusion tensor field $\mathbf{K}$ used for SR-PDE. Right: triangulation of the domain used for SSR and SR-PDE.

on the entire domain;

B. $n=100$ observation points sampled only on the first and third quadrants;

C. $n=100$ observation points sampled in a crossshape pattern.

The experiment is replicated 50 times. For each study case, A, B, and C, and each replicate: we sample the location points, $\mathbf{p}_{1}, \ldots, \mathbf{p}_{n}$; we sample independent errors, $\epsilon_{1}, \ldots, \epsilon_{n}$, from a Gaussian distribution with mean 0 and standard deviation $\sigma=0.1$; we thus obtain observations $z_{1}, \ldots, z_{n}$ from model (1) with the true function $f_{0}$ displayed in Figure 7.

The surface $\hat{f}$ is estimated using three methods:

1. SR-PDE (anisotropic and non-stationary smoothing);

2. standard SSR (isotropic and stationary smoothing);

\section{SOAP (isotropic and stationary smoothing).}

For all the three methods, we impose homogeneous Dirichlet b.c., $\left.f\right|_{\partial \Omega}=0$; for each simulation study, each replicate and each method, the value of the smoothing parameter $\lambda$ is chosen via GCV.

The triangulation used for the SR-PDE and standard SSR estimation is a uniform mesh on the domain, represented in Figure 7 Right, with approximately 100 vertices. Both for SR-PDE and SSR we use a linear Finite Element space for the discretization of the surface estimator.

Using SR-PDE it is possible to incorporate the prior knowledge on the shape of the surface, that should have almost circular isolines. We can achieve this by penalizing a PDE that smooths the surface along concentric circles; specifically we consider the anisotropic and non-stationary diffusion tensor illustrated in the central panel of Figure 7 and given by

$$
\begin{aligned}
\mathbf{K}(x, y) & =\left[\begin{array}{cc}
y^{2}+\kappa_{1} x^{2} & \left(\kappa_{1}-1\right) x y \\
\left(\kappa_{1}-1\right) x y & x^{2}+\kappa_{1} y^{2}
\end{array}\right] \\
& +\kappa_{2}\left(R^{2}-x^{2}-y^{2}\right) \mathbf{I}_{2},
\end{aligned}
$$

where $R$ denotes the largest radius in this almost circular domain (in the simulations, $R=1$ ). The first hyperparameter in (20), $\kappa_{1}$, represents the ratio between the diffusion in the radial and in the circular 
CASE A

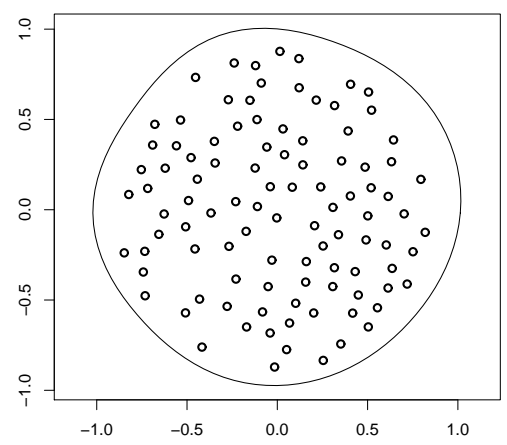

SSR

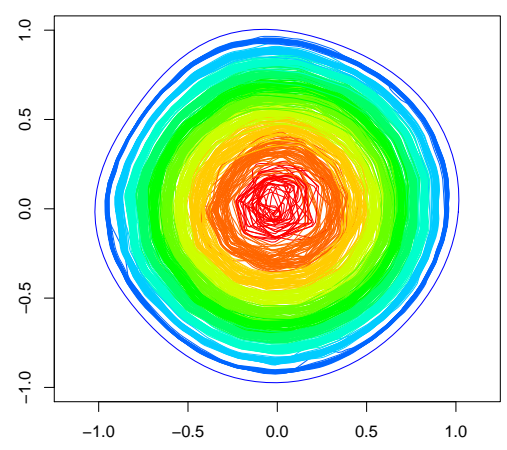

SR-PDE

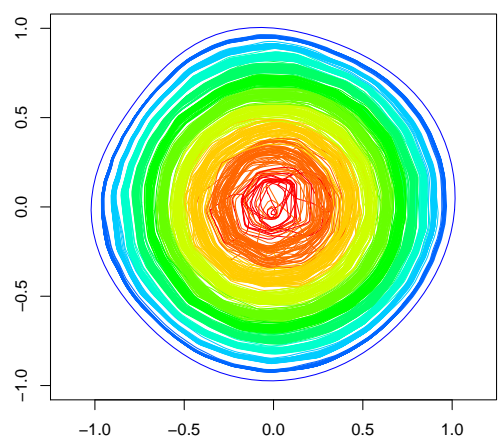

SOAP

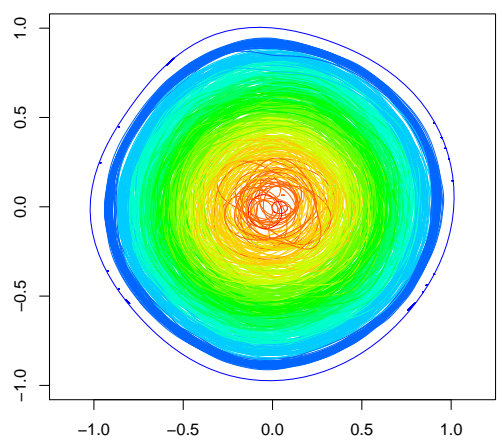

Figure 8: Top left: location points sampled in the first replicate for case A. Top right, bottom left, bottom right: surface estimates obtained using respectively SR-PDE, SSR and SOAP; the images display the isolines $(0,0.1, \ldots, 0.9,1)$ of the surface estimates obtained in the 50 simulation replicates; the isolines are colored using the same color scale used for the isolines of the true function $f_{0}$ in Figure 7.

direction, and we set $\kappa_{1}=0.01$. The anisotropic and non-stationary part of the diffusion field, which corresponds to the first term of the right-hand side of (20), is stronger near the boundary and completely vanishes in the center of the carotid; instead the isotropic and stationary part, which corresponds to the second term of the right-hand side of (20), vanishes near the boundary. The relative strength of the stationary isotropic and non-stationary anisotropic part is controlled via the second hyperparameter $\kappa_{2}$, and we set $\kappa_{2}=0.1$. The transport field, the reaction term and the forcing term are set equal to zero, i.e., $\mathbf{b}=0, c=0$ and $u=0$.
Standard SSR instead is not able to take advantage of the specific prior knowledge of the shape of the surface, and enforces an isotropic and stationary smoothing, corresponding to SR-PDE with $\mathbf{K}=\mathbf{I}$, $\mathbf{b}=0, c=0$ and $u=0$. Also SOAP produces an isotropic and stationary smoothing; this technique is implemented using the function gam, in the R package mgcv 1.7-22, see Wood (2011), using 49 interior knots on a lattice.

Figures 8-10 show the results obtained using the different methods in the three considered scenarios, cases A, B and C. The upper left panel of the figures shows the location points sampled in the first repli- 
CASE B

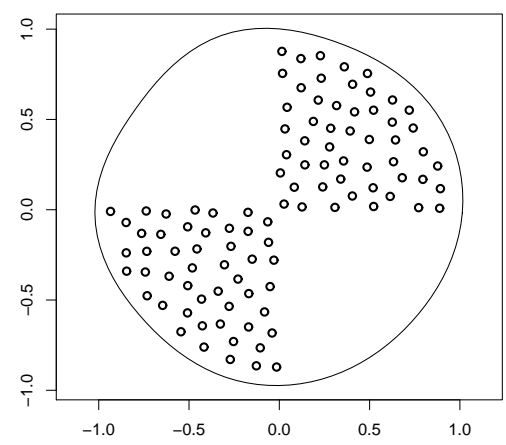

SSR

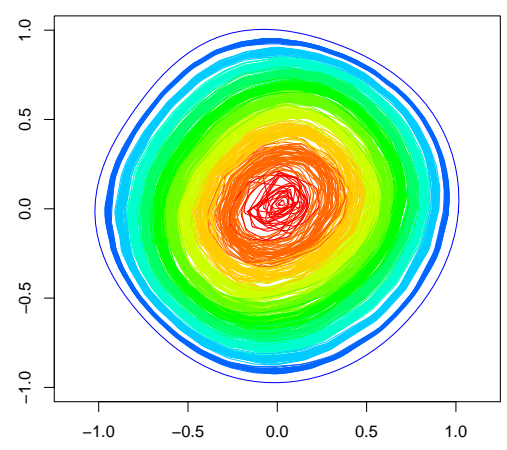

SR-PDE

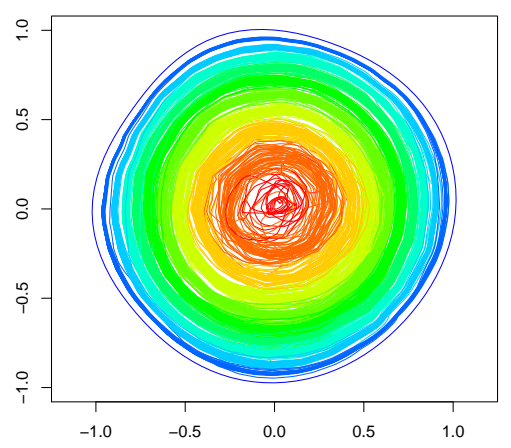

SOAP

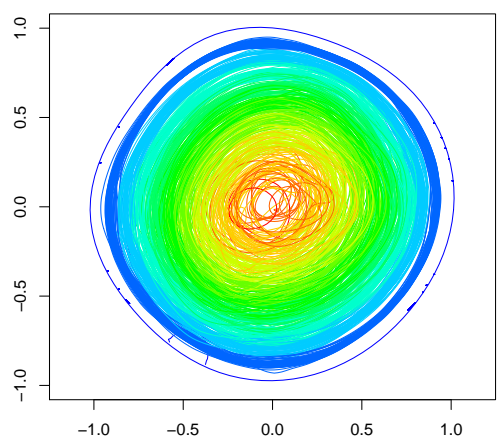

Figure 9: Same as Figure 8, for case B.

cate in each of the three different scenarios. The top right, bottom left, bottom right panels of these figures display the surface estimates obtained using respectively SR-PDE, SSR and SOAP. In particular, the images display the isolines $(0,0.1, \ldots, 0.9,1)$ of the surface estimates obtained in the 50 simulation replicates; the isolines are colored using the same color scale used for the isolines of the true function $f_{0}$ in Figure 7. These images highlight some aspects of the bias and variance of the surface estimators. The shape of the estimated surfaces emphasize the main bias aspects of the estimators, while the dispersion or concentration of the isolines highlights respectively the larger or smaller variability of the estimator.

Comparing the results obtained with the three methods we can notice that the inclusion of the prior knowledge improves the estimate, especially when data are distributed only on subregions of the domain. We can in fact see that in the three case studies the surfaces estimated with SR-PDE smoothing have circular isolines similar to those of the true surface $f_{0}$. Instead, when the prior knowledge is not included in the model, i.e., for standard SSR and SOAP, the surface estimates tend to depend on the design of the experiments. We notice in fact that the isolines of SSR and SOAP estimates are similar to ellipses in case B and to rhomboids in case $\mathrm{C}$, instead of circles. This is due to the fact that both methods tend to fit planes in those areas where no observations are available. This phenomenon is more appar- 
CASE C

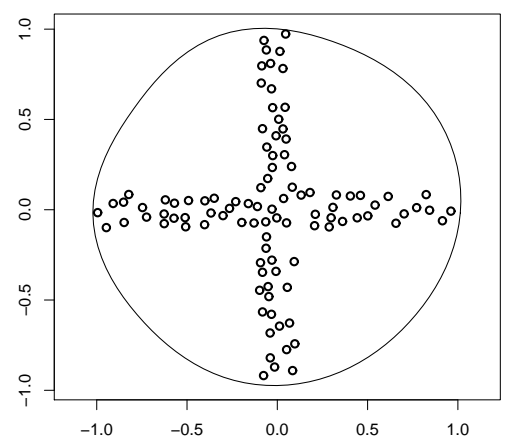

SSR

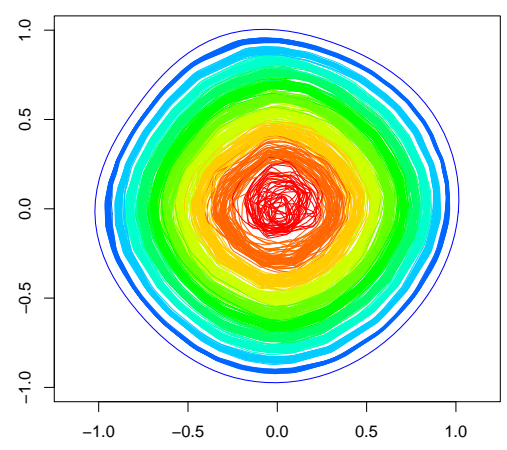

SR-PDE

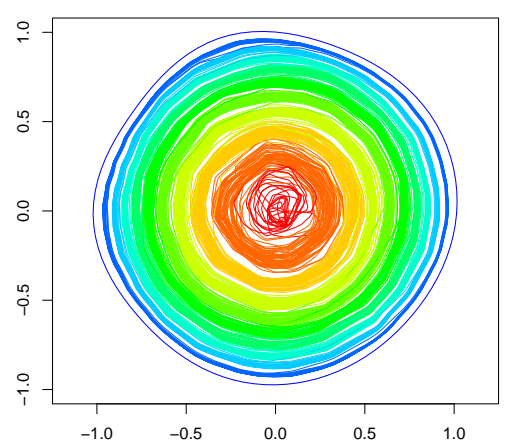

SOAP

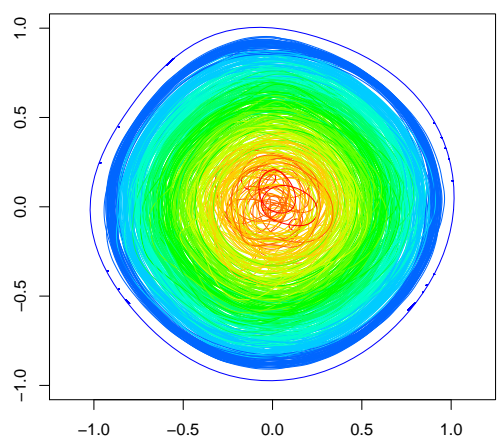

Figure 10: Same as Figure 8, for case C.

ent with SSR than with SOAP because SOAP esti- 8-10.

mates have an higher variability.

Figure 11 shows the comparison of the three methods in terms of Root Mean Square Error (RMSE) of the corresponding estimators, with the RMSE evaluated on a fine lattice of step 0.01 over the domain $\Omega$. The boxplots highlight that incorporation of the prior knowledge on the shape of the surface leads to a large improvement in the estimation. SR-PDE smoothing provides in fact significantly better estimates of $f_{0}$ than the other two methods. The boxplots also show that SR-PDE estimates display lower variability than SSR and SOAP estimates. This phenomenon is also visible from the isolines of the estimated surfaces with SR-PDE, SSR and SOAP represented Figures
The simulation studies reported in the supplementary material show that incorporating the prior knowledge on the phenomenon leads to significantly improved estimates also in the areal data framework.

\section{Application to the blood-flow ve- locity field estimation}

Carotid ECD is usually the first imaging procedure used to diagnose carotid artery diseases, such as ischemic stroke, caused by the presence of an atherosclerotic plaque. ECD data in our study have been collected using a Diagnostic Ultrasound System Philips iU22 (Philips Ultrasound, Bothell, U.S.A.) 

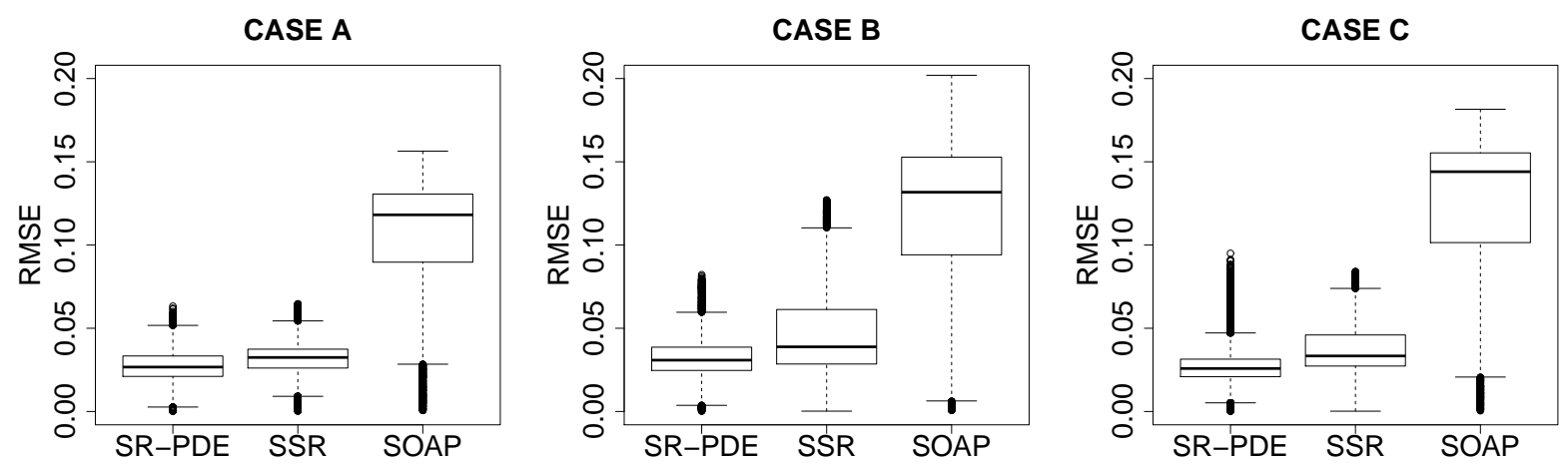

Figure 11: Boxplot of RMSE (evaluated on a fine lattice of step 0.01 over the domain $\Omega$ ) for SR-PDE, SSR and SOAP estimators, in case studies A, B and C.

with a L12-5 probe. The septum that divides the carotid bifurcation is localized and marked as a reference point. With the help of an electronic rule, we localize the other points of acquisition of the blood velocity; specifically, in our protocol the blood flow velocity is measured in standard locations points, according to the cross-shaped design represented in Figure 2, on the carotid cross-section located $2 \mathrm{~cm}$ before the reference point indicated above.

In order to estimate the systolic velocity field on this cross-section of the carotid we minimize the functional $\bar{J}(f)$ defined in (8). As mentioned in Section 1 we know that a physiological velocity profile has smooth and almost circular isolines. For this reason we choose to penalize a PDE that includes the non-stationary anisotropic diffusion tensor shown in the left panel of Figure 4 and described in equation (20), that smooths the observations in the tangential direction of concentric circles. The largest section radius is $R=2.8$; we set $\kappa_{1}=0.1$, in order to impose a smoothing effect that is one order of magnitude stronger in the tangential direction of circles than in the radial direction, and $\kappa_{2}=0.2$, to impose an amount of isotropic smoothing in the center of the vessel that is comparable to the anisotropic smoothing in the periphery of the vessel. Moreover, we also know that, due to viscosity of the blood, a physiological velocity field is rather flat on the central part of the artery lumen. For this reason, we also include in the PDE model the non-stationary transport field shown in the right panel of Figure 4, which smooths the observations in the radial direction, from the center of the cross-section to the boundary: $\mathbf{b}(x, y)=$ $(\beta x, \beta y)^{T}$, where the hyperparameter $\beta$ represents the intensity of the transport field. This transport term in fact penalizes high first derivatives in the radial direction, providing velocity profiles that tend to flatten in the central part of the artery lumen. Here we set $\beta=0.5$ to impose a smoothing effect that is comparable to the anisotropic smoothing induced by the diffusion term. The reaction parameter and the forcing term are not needed in this application, hence we set $c=0$ and $u=0$. Finally, we know that blood flow velocity is zero at the arterial wall, due to friction between the blood particles and the vessel wall (the above mentioned no-slip conditions); hence we impose homogeneous Dirichlet b.c.: $\left.f\right|_{\partial \Omega}=0$. The problem is then discretized by means of linear Finite 
Elements defined on the mesh represented in Figure 5.

Figure 12 displays the velocity field estimated using SR-PDE smoothing. The maximum estimated velocity is $31.75 \mathrm{~cm} / \mathrm{s}$ with an estimated standard deviation $\hat{\bar{\sigma}}=1.92 \mathrm{~cm} / \mathrm{s}$. A visual comparison with the estimate obtained for the same data by standard SSR, shown in Figure 3, immediately highlights the advantages of the proposed technique. Whilst the standard SSR estimate is strongly influenced by the cross-shaped pattern of the observations and displays strongly rhomboidal isolines, forcing the surface estimate towards a plane in regions where no observations are available, the SR-PDE efficiently uses the a priori information on the phenomenon under study and returns a realistic estimate of the blood flow, which is not affected by the cross-shaped pattern of the observations and displays physiological and smooth isolines.

Notice that the SR-PDE estimate in Figure 12 captures an asymmetry in the data, resulting in an eccentric estimate of the blood flow: the velocity peak is in fact not in the center of the cross-section but in the lower part where higher velocities are measured. This feature of the blood flow is indeed justified by the curvature of the carotid artery and by the non-stationarity of the blood flow. SR-PDE estimates in fact accurately highlight important features of the blood flow, such as eccentricity, asymmetry and reversion of the fluxes, that are of interest to the medical doctors, in order to understand how the local hemodynamics influences atherosclerosis pathogenesis. As mentioned in the Introduction, MACAREN@MOX project aims at exploring this relationship, investigating how different hemo-

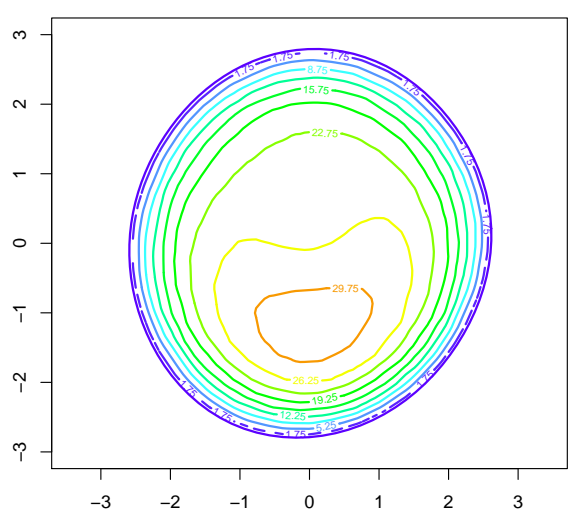

Figure 12: Estimate of the blood-flow velocity field in the carotid section using SR-PDE.

dynamical patterns affect the plaque formation process. For this reason, obtaining accurate physiological estimates of blood flow velocity fields is a first crucial goal of the project. Indeed, the SR-PDE estimates will then be used in population studies that compare the blood flow velocity field in patients vs healthy subjects, and that compare the velocity field in patients before and after the removal of the carotid plaque via thromboendarterectomy. Notice that such population studies involve the comparisons of estimates referred to different domains, since the cross-sections of the carotids have patient-specific shapes; to face this issue we are currently developing an appropriate registration method (see Section 8) and these analyses will be the object of a following dedicated work. The estimated velocity fields will also be used as inflow conditions for the hemodynamic simulations performed using patient-specific carotid morphologies. The prescription of suitable inflow conditions in computational fluid-dynamics is in fact a major issue; see, e.g., Veneziani and Vergara 
(2005). Moreover, the computation of the variance of the surface estimator will also be used to investigate the sensitivity of these simulations to the specified inflow conditions and will provide some understanding on how their misspecification affects the results. These numerical simulations will in turn offer enhanced data that give a richer information on hemodynamical regimes in the carotid bifurcation, further allowing the study of its impact on atherosclerosis. Computational fluid-dynamic simulations are also of great interest because they allow to synthetically verify the impact of different surgical interventions, evaluating which one is more prone to the reformation of the plaque or to other complications. In the future, this could become an important tool for comparing beforehand the effects of different interventions for a given patient, considering the geometry of the patient carotid and the properties of the atherosclerotic plaque, thus giving important suggestions to clinicians on the surgical operation to choose in the specific patient case.

\section{Conclusion and future work}

In this work we have introduced an innovative method for surface and spatial field estimation, when prior knowledge is available, concerning the physics of the problem. In particular, this prior knowledge, conveniently described via a PDE, is used to model the space variation of the phenomenon. Although demonstrated on the specific application that motivated its development, the method has indeed a very broad applicability, since PDEs are commonly used to model phenomena behavior in many fields of sciences and engineering.

One of the most interesting developments within this line of research consists now in the data driven estimation of the hyperparameters in the penalized PDE. In the current study, these hyperparameters have in fact been considered fixed. Notice that, while a currently crucial topic in statistics concerns the development of methods for parameter estimations in Ordinary Differential Equation, this would instead consist in approaching the remarkably more complex problem of data driven estimation of the parameters in PDEs. Although this research field is subject of specific interest by the inverse problem community (see, e.g., Stuart (2010)), we believe that statisticians have not yet devoted much attention to this problem. Within the considered framework, a possible road to tackle this problem is offered for instance by the parameter cascading methodology proposed in Ramsay et al. (2007). A first promising attempt to apply this methodology to the parameter estimation in PDEs is provided in Xun et al. (2013).

As derived in Section 4, the proposed estimators are linear in the observed data values and have a typical penalized regression form, so that important distributional properties can be readily derived. We are currently also studying the (infill) asymptotic properties of these estimators, when the number of observations $n$ goes to infinity and the characteristic mesh size $h$ goes to zero. The numerical convergence of the estimator when $h$ goes to zero is detailed in Azzimonti et al. (2014).

The proposed method can also be extended to include the time dimension, in order to model surfaces evolving in time. Such extension would allow to study how the blood-flow velocity field varies during the time of the heart beat. Notice that it is necessary in this case to allow for changes of the shape of the 
domain over time, to account for the deformation of

the artery wall during the heart beat. This poses a problem of registration of different domains similar to the one faced in population studies (see Section 7).

Finally, the method could also be extended to Riemannian manifold domains, by appropriately setting the problem in the framework presented in Ettinger et al. (2012).

\section{References}

Azzimonti, L., Nobile, F., Sangalli, L., and Secchi, P. (2014), "Mixed Finite Elements for spatial regression with PDE penalization," SIAM-ASA Journal on Uncertainty Quantification, to appear.

Buja, A., Hastie, T., and Tibshirani, R. (1989), "Linear smoothers and additive models," Annals of Statististics, 17, 153-555.

D’Elia, M., Perego, M., and Veneziani, A. (2012), “A Variational Data Assimilation Procedure for the Incompressible NavierStokes Equations in Hemodynamics," Journal of Scientific Computing, 52, 340-359.

D'Elia, M. and Veneziani, A. (2012), "Uncertainty quantification for data assimilation in a steady incompressible NavierStokes problem," ESAIM Mathematical Modelling and Numerical Analysis, 47, 1037-1057.

Ettinger, B., Perotto, S., and Sangalli, L. (2012), "Spatial regression models over two-dimensional manifolds," Tech. Rep. 54/2012, MOX - Dipartimento di Matematica, Politecnico di Milano, available at http://mox.polimi.it/it/progetti/pubblicazioni/.

Evans, L. (1998), Partial Differential Equations, American Mathematical Society.

Formaggia, L., Quarteroni, A., and Veneziani, A. (2009), Cardiovascular mathematics: modeling and simulation of the circulatory system, Springer.

Guillas, S. and Lai, M. (2010), "Bivariate splines for spatial functional regression models," J. Nonparametric Stat, 22, 477-497.

Hastie, T. and Tibshirani, R. (1990), Generalized additive models, London: Chapman and Hall Ltd.

Hinze, M., Pinnau, R., Ulbrich, M., and Ulbrich, S. (2009), Optimization with PDE Constraints, Springere Berlin.
Lindgren, F., Rue, H., and Lindström, J. (2011), “An explicit link between Gaussian fields and Gaussian Markov random fields: the stochastic partial differential equation approach," J. R. Stat. Soc. Ser. B Stat. Methodol., 73, 423-498.

Lions, J. (1971), Optimal control of systems governed by partial differential equations, Springer.

Moyle, K., Antiga, L., and Steinman, D. (2006), "Inlet conditions for image-based CFD models of the carotid bifurcation: is it reasonable to assume fully developed flow?" J Biomech Eng., 128, 371-379.

Pironneau, O., Hecht, F., Le Hyaric, A., and Morice, J. (2011), FreeFem++ Software version 3.16, available at www.freefem.org.

Quarteroni, A. (2008), Numerical models for differential problems, Springer.

R Development Core Team (2012), R: A Language and Environment for Statistical Computing, R Foundation for Statistical Computing, available at www.R-project.org.

Ramsay, J., Hooker, G., Campbell, D., and Cao, J. (2007), "Parameter estimation for differential equations: a generalized smoothing approach," J. R. Stat. Soc. Ser. B Stat. Methodol., 69, 741-796.

Ramsay, T. (2002), "Spline Smoothing over Difficult Regions," J. R. Stat. Soc. Ser. B Stat. Methodol., 64, 307-319.

Rozza, G., Manzoni, A., and Negri, F. (2012), "Reduction strategies for PDE-constrained optimization problems in haemodynamics," Proceedings of ECCOMAS 2012, European Congress on Computational Methods in Applied Sciences and Engineering.

Sangalli, L., Ramsay, J., and Ramsay, T. (2013), "Spatial spline regression models," J. R. Stat. Soc. Ser. B Stat. Methodol., 75, 681-703.

Stuart, A. (2010), "Inverse problems: a Bayesian perspective," Acta Numerica, 19, 451-559.

Veneziani, A. and Vergara, C. (2005), "Flow rate defective Boundary Conditions in Haemodynamics Simulations," Int. Journ. Num. Meth. Fluids, 47, 803-816.

Wood, S. (2011), "Fast stable restricted maximum likelihood and marginal likelihood estimation of semiparametric generalized linear models," J. R. Stat. Soc. Ser. B Stat. Methodol., 73, 3-36.

Wood, S., Bravington, M., and Hedley, S. (2008), "Soap film smoothing," J. R. Stat. Soc. Ser. B Stat. Methodol., 70, 931955. 
Xun, X., Cao, J., Mallick, B., Maity, A., and Carroll, R. (2013), "Parameter Estimation of Partial Differential Equation Models," Journal of the American Statistical Association, 108, 1009-1020.

\section{A Assumptions and proof of Propo- sition 1}

In order to prove the existence and the uniqueness of the surface estimator we need to require some regularity to the parameters of the PDE, see, e.g., Evans (1998).

Assumption 1. The parameters of the PDE should be such that the solution of the second order elliptic PDE exists and is unique. A set of sufficient conditions is: $K_{i j}, b_{j}, c \in L^{\infty}(\Omega)$, $\mathbf{K}$ is symmetric and uniformly elliptic, i.e., $\boldsymbol{\xi}^{T} \mathbf{K}(\mathbf{x}) \boldsymbol{\xi} \geq \alpha_{K}$ $\forall \mathbf{x} \in \Omega$ and $\forall \boldsymbol{\xi} \in \mathbb{R}^{d},-1 / 2 \operatorname{div}(\mathbf{b}(\mathbf{x}))+c(\mathbf{x}) \geq-\alpha_{K} / C_{P}$, where $\alpha_{K}$ is the ellipticity constant and $C_{P}$ is the so called Poincaré constant such that $\|v\|_{L^{2}(\Omega)} \leq C_{P}\|\nabla v\|_{L^{2}(\Omega)}$ for any $v \in H^{1}(\Omega)$ vanishing on some portion of the boundary. Moreover we require that $K_{i j}$ is Lipschitz continuous, so that the solution is in $V$.

Existence and uniqueness of the estimator $\hat{f}$ are obtained thanks to the classical theorem stated, e.g., in Lions (1971):

Theorem 1. Consider the functional

$$
J(g)=\mathcal{A}(g, g)+\mathcal{L} g+c
$$

where $\mathcal{A}: \mathcal{G} \times \mathcal{G} \rightarrow \mathbb{R}$ is a continuous, coercive and symmetric bilinear form in $\mathcal{G}$, i.e., $\mathcal{A}(g, \varphi) \leq M\|g\|_{\mathcal{G}}\|\varphi\|_{\mathcal{G}}, \mathcal{A}(g, g) \geq$ $\alpha\|g\|_{\mathcal{G}}^{2}$ and $\mathcal{A}(g, \varphi)=\mathcal{A}(\varphi, g) \forall g, \varphi \in \mathcal{G}, \mathcal{L}: \mathcal{G} \rightarrow \mathbb{R}$ is a linear operator, $c$ is a constant and $\mathcal{G}$ is a Hilbert space. Then there exists a unique $\hat{g} \in \mathcal{G}$ such that $J(\hat{g})=\inf _{\mathcal{G}} J(g)$. Moreover $\hat{g}$ satisfies the following equation:

$$
\left(J^{\prime}(\hat{g}), \varphi\right)=2 \mathcal{A}(\hat{g}, \varphi)+\mathcal{L} \varphi=0 \forall \varphi \in \mathcal{G} .
$$

Proof of Proposition 1. We introduce a new quantity $g \in$ $L^{2}(\Omega)$, defined as $g=L f-u$, which represents the misfit of the PDE in the penalizing term, and the operator $B: L^{2}(\Omega) \rightarrow V$, defined as the inverse of the operator $L$, i.e., $L(B \tilde{u})=\tilde{u}$ in $\Omega$ and $B \tilde{u}=0$ on $\partial \Omega$. Under the regularity conditions required for the operator $L$, classical results of analysis (see, e.g., Evans (1998)) state the existence and the uniqueness of the operator $B$ and provide a bound for the quantity $B \tilde{u}$ :

$$
\|B u\|_{H^{2}(\Omega)} \leq C\|u\|_{L^{2}(\Omega)} .
$$

Thanks to the definition of $g$ and $B$ we can write $f$ as an affine transformation of $g$, i.e., $f=B(u+g)$, and the functional (2) as

$$
\begin{aligned}
J_{g}(g)=J(B(u+g)) & =\sum_{i=1}^{n}\left(B(u+g)\left(\mathbf{p}_{i}\right)-z_{i}\right)^{2} \\
& +\lambda\|g\|_{L^{2}(\Omega)}^{2}
\end{aligned}
$$

This reformulation of the functional $J$ is very useful since we can now write $J_{g}$ in the quadratic form (21) where $\mathcal{G}=L^{2}(\Omega)$,

$$
\begin{aligned}
\mathcal{A}(g, \varphi) & =\sum_{i=1}^{n} B g\left(\mathbf{p}_{i}\right) B \varphi\left(\mathbf{p}_{i}\right)+\lambda \int_{\Omega} g \varphi \\
\mathcal{L} \varphi & =2 \sum_{i=1}^{n} B \varphi\left(\mathbf{p}_{i}\right)\left(B u\left(\mathbf{p}_{i}\right)-z_{i}\right) \\
c & =\sum_{i=1}^{n}\left(B u\left(\mathbf{p}_{i}\right)-z_{i}\right)^{2} .
\end{aligned}
$$

Clearly $\mathcal{A}(g, \varphi)$ is a bilinear form, since both $B$ and the pointwise evaluation of a function are linear operators. Moreover, it is continuous in $L^{2}(\Omega)$; indeed, thanks to the embedding $H^{2}(\Omega) \subset C(\bar{\Omega})$ if $\Omega \subset \mathbb{R}^{d}$ with $d \leq 3$ and thanks to (23) we have that $\left|B g\left(\mathbf{p}_{i}\right)\right| \leq\|B g\|_{C(\bar{\Omega})} \leq$ $C\|B g\|_{H^{2}(\Omega)} \leq C\|g\|_{L^{2}(\Omega)}$. We thus obtain that $\mathcal{A}(g, \varphi) \leq$ $\left(C^{2}+\lambda\right)\|g\|_{L^{2}(\Omega)}\|\varphi\|_{L^{2}(\Omega)}$. Finally, the operator $\mathcal{A}(g, \varphi)$ is coercive in $L^{2}(\Omega)$, since

$$
\mathcal{A}(g, g)=\sum_{i=1}^{n}\left|B g\left(\mathbf{p}_{i}\right)\right|^{2}+\lambda \int_{\Omega} g^{2} \geq \lambda \int_{\Omega} g^{2}=\lambda\|g\|_{L^{2}(\Omega)}^{2} .
$$

Due to the fact that the bilinear form $\mathcal{A}(\cdot, \cdot)$ is continuous and coercive in $L^{2}(\Omega)$, that the operator $\mathcal{L}$ is linear and that $c$ is a constant, Theorem 1 states the existence and the uniqueness of $\hat{g}=\operatorname{argmin}_{g \in L^{2}(\Omega)} J_{g}(g)$. We can thus deduce the existence and uniqueness of $\hat{f}=B(\hat{g}+u)=\operatorname{argmin}_{f \in V} J(f)$.

Thanks to Theorem 1 and the definition of the operator $B$ we can obtain the surface estimator $\hat{f}$ as the solution of the PDE

$$
\begin{cases}L \hat{f}=u+\hat{g} & \text { in } \Omega \\ \hat{f}=0 & \text { on } \partial \Omega\end{cases}
$$

where $\hat{g}$ is obtained as the solution of the equation (22), that can be written as

$$
\begin{aligned}
\frac{1}{2}\left(J_{g}^{\prime}(\hat{g}), \varphi\right) & =\int_{\Omega}\left[\sum_{i=1}^{n}\left(\hat{f}-z_{i}\right) \delta_{\mathbf{p}_{i}}+\lambda L^{*} \hat{g}\right] B \varphi \\
& +\int_{\partial \Omega} \hat{g} B \varphi=0 \forall \varphi \in \mathcal{G} .
\end{aligned}
$$

This equation corresponds to the PDE

$$
\begin{cases}L^{*} \hat{g}=-\frac{1}{\lambda} \sum_{i=1}^{n}\left(\hat{f}-z_{i}\right) \delta_{\mathbf{p}_{i}} & \text { in } \Omega \\ \hat{g}=0 & \text { on } \partial \Omega\end{cases}
$$

where $\delta_{\mathbf{p}_{i}}$ is the Dirac mass located in $\mathbf{p}_{i}, L^{*}$ is the adjoint operator of $L$, defined in (5). The surface estimator can thus be written as the solution of a coupled system of the second order PDEs (24) and (25). It should be noticed that solving this coupled system of PDEs is indeed equivalent to solving the fourth 
order problem

$$
\left(L^{*} L+\frac{1}{\lambda} \sum_{i=1}^{n} \delta_{\mathbf{p}_{i}}\right) \hat{f}=L^{*} u+\frac{1}{\lambda} \sum_{i=1}^{n} z_{i} \delta_{\mathbf{p}_{i}}
$$

that can be obtained integrating by parts the penalty term in the functional $J(f)$ and deriving the functional with respect to $f$. Solving the coupled system of second order PDEs is however more convenient than solving a fourth order problem since it is possible to use classical numerical techniques to discretize the problem.

The same strategy used in order to prove Proposition 1 can be used in the proof of Proposition 2, which is moreover simplified by the presence of areal data in the latter case, instead of pointwise data. It is in fact not necessary to require that the solution is a continuous function, since we are interested only in integral quantities. When working with areal data it is possible to relax the Assumption 1, not requiring that $K_{i j}$ is Lipschitz continuous.

\section{B The penalization matrix}

Assume for the moment that $\psi_{j}$ are smooth functions and neglect the forcing term $u$. The penalty matrix $\mathbf{P}$ in (16) can be written as

$$
\mathbf{P}=\int_{\Omega} \int_{\Omega} L \boldsymbol{\psi}(\mathbf{s}) \boldsymbol{\psi}^{T}(\mathbf{s})\left[\int_{\Omega} \boldsymbol{\psi} \boldsymbol{\psi}^{T}\right]^{-1} \boldsymbol{\psi}(\mathbf{t}) L \boldsymbol{\psi}^{T}(\mathbf{t}) d \mathbf{s} d \mathbf{t}
$$

where $L \boldsymbol{\psi}=\left(L \psi_{1}, \ldots, L \psi_{N_{h, 0}}\right)^{T}$, since $\mathbf{A}_{i j}=a\left(\psi_{j}, \psi_{i}\right)=$ $\int_{\Omega} \psi_{i} L \psi_{j}$. The matrix $\tilde{\mathbf{P}}=\int_{\Omega} L \boldsymbol{\psi} L \boldsymbol{\psi}^{T}$, which may instead be obtained as direct discretization of the penalty term in (2), can be represented as

$$
\tilde{\mathbf{P}}=\int_{\Omega} \int_{\Omega} L \boldsymbol{\psi}(\mathbf{s}) \delta(\mathbf{s}, \mathbf{t}) L \boldsymbol{\psi}^{T}(\mathbf{t}) d \mathbf{s} d \mathbf{t}
$$

using the kernel operator associated with the $L^{2}$ space, $\delta(\mathbf{s}, \mathbf{t})$, defined as

$$
\int_{\Omega} \delta(\mathbf{s}, \mathbf{t}) q(\mathbf{t}) d \mathbf{t}=q(\mathbf{s}) \forall q \in L^{2}(\Omega) \cap C(\Omega) .
$$

From the above equations we see that $\mathbf{P}$ is an approximation in a weak sense of $\tilde{\mathbf{P}}$. In fact, the operator $\delta(\mathbf{s}, \mathbf{t})$ is approximated, in the mixed Finite Element approach here considered, with the projection operator

$$
\boldsymbol{\psi}^{T}(\mathbf{s})\left[\int_{\Omega} \boldsymbol{\psi} \boldsymbol{\psi}^{T}\right]^{-1} \boldsymbol{\psi}(\mathbf{t})
$$

that projects functions on $\operatorname{span}\left\{\psi_{1}, \ldots, \psi_{N_{h, 0}}\right\}$. This operator satisfies the property (26) in $\operatorname{span}\left\{\psi_{1}, \ldots, \psi_{N_{h}}\right\}$; in fact, if $q(\mathbf{t})=\sum_{k=1}^{K} q_{k} \psi_{k}(\mathbf{t})$, then

$$
\int_{\Omega} \boldsymbol{\psi}^{T}(\mathbf{s})\left[\int_{\Omega} \boldsymbol{\psi} \boldsymbol{\psi}^{T}\right]^{-1} \boldsymbol{\psi}(\mathbf{t}) q(\mathbf{t}) d \mathbf{t}=\sum_{k=1}^{K} q_{k} \psi_{k}(\mathbf{s})=q(\mathbf{s})
$$

while if $q \notin \operatorname{span}\left\{\psi_{1}, \ldots, \psi_{N_{h, 0}}\right\}$ this operator projects the function $q$ on $\operatorname{span}\left\{\psi_{1}, \ldots, \psi_{N_{h, 0}}\right\}$.

\section{General boundary conditions}

In the case of general boundary conditions, the space $V$ is the space of functions in $L^{2}(\Omega)$ with first and second derivatives in $L^{2}(\Omega)$ that satisfy (19).

Starting with the pointwise data framework, the estimation problem with general b.c. (19) is analogous to Problem 1. The existence and uniqueness of the surface estimator $\hat{f}$ is proved in the case of general boundary conditions analogously to the case of homogeneous Dirichlet b.c. detailed in Appendix A. The unique solution of the problem, $\hat{f} \in V$, is obtained by solving

$$
\begin{aligned}
& \begin{cases}L \hat{f}=u+\hat{g} & \text { in } \Omega \\
+ \text { b.c. } & \text { on } \partial \Omega\end{cases} \\
& \begin{cases}L^{*} \hat{g}=-\frac{1}{\lambda} \sum_{i=1}^{n}\left(\hat{f}-z_{i}\right) \delta_{\mathbf{p}_{i}} & \text { in } \Omega \\
+ \text { b.c. } & \text { on } \partial \Omega\end{cases}
\end{aligned}
$$

where b.c. ${ }^{*}$ are the boundary conditions associated to the adjoint problem, i.e.,

$$
\begin{cases}g=0 & \text { on } \Gamma_{D} \\ \mathbf{K} \nabla g \cdot \boldsymbol{\nu}+\mathbf{b} \cdot \boldsymbol{\nu} g=0 & \text { on } \Gamma_{N} \\ \mathbf{K} \nabla g \cdot \boldsymbol{\nu}+(\mathbf{b} \cdot \boldsymbol{\nu}+\chi) g=0 & \text { on } \Gamma_{R}\end{cases}
$$

Notice that these conditions are always homogeneous.

We define now the space $V_{h, \Gamma_{D}}^{r}$ of continuous piecewise polynomial functions of degree $r \geq 1$ on the domain triangulation, that vanish on $\Gamma_{D}$, the part of the boundary $\partial \Omega$ with Dirichlet b.c. (if $\Gamma_{D}$ is not empty):

$V_{h, \Gamma_{D}}^{r}=\left\{v \in C^{0}(\bar{\Omega}):\left.v\right|_{\Gamma_{D}}=0\right.$ and $\left.\left.v\right|_{\boldsymbol{\tau}} \in \mathbb{P}^{r}(\boldsymbol{\tau}) \forall \boldsymbol{\tau} \in \mathcal{T}_{h}\right\}$.

We denote by $\psi_{1}, \ldots, \psi_{N_{h, \Gamma_{D}}}$, where $N_{h, \Gamma_{D}}=\operatorname{dim}\left(V_{h, \Gamma_{D}}^{r}\right)$, the Finite Element basis functions of this space, and by $\boldsymbol{\xi}_{1}, \ldots, \boldsymbol{\xi}_{N_{h, \Gamma_{D}}}$ the associated nodes; note that the basis now include the basis functions associated with the internal nodes and with the nodes on $\Gamma_{N}$ and $\Gamma_{R}$.

System (27) is solved in a different way if the Dirichlet b.c. are homogeneous $\left(\gamma_{D}=0\right)$ or not $\left(\gamma_{D} \neq 0\right)$.

If the Dirichlet b.c. are homogeneous or there are no Dirichlet b.c. (i.e., $\Gamma_{D}$ is empty), the discretization of the variational formulation associated to (27) is

$\left\{\begin{array}{l}a\left(\hat{f}_{h}, \psi_{h}\right)-\int_{\Omega} \hat{g}_{h} \psi_{h}=\int_{\Omega} u \psi_{h}+\int_{\Gamma_{N}} \gamma_{N} \psi_{h}+\int_{\Gamma_{R}} \gamma_{R} \psi_{h} \\ \lambda a\left(\varphi_{h}, \hat{g}_{h}\right)+\sum_{i=1}^{n} \hat{f}_{h}\left(\mathbf{p}_{i}\right) \varphi_{h}\left(\mathbf{p}_{i}\right)=\sum_{i=1}^{n} z_{i} \varphi_{h}\left(\mathbf{p}_{i}\right)\end{array}\right.$

for all $\psi_{h}, \varphi_{h} \in V_{h, \Gamma_{D}}^{r}$, where the bilinear form $a(\cdot, \cdot)$ is now defined as

$a(\hat{f}, \psi)=\int_{\Omega}(\mathbf{K} \nabla \hat{f} \cdot \nabla \psi+\mathbf{b} \cdot \nabla \hat{f} \psi+c \hat{f} \psi)+\int_{\Gamma_{R}} \chi \hat{f} \psi$

Consider the matrices $\mathbf{R}_{x x}(\mathbf{K}), \mathbf{R}_{x y}(\mathbf{K}), \mathbf{R}_{y y}(\mathbf{K}), \mathbf{R}_{x}(\mathbf{b})$, $\mathbf{R}_{y}(\mathbf{b}), \mathbf{R}(c)$ defined in Section 4.1 , and the matrix $\boldsymbol{\Psi}$ of ba- 
sis evaluations (15), where now $\boldsymbol{\psi}=\left(\psi_{1}, \ldots, \psi_{N_{h, \Gamma_{D}}}\right)^{T}$, and define the matrix

$$
\mathbf{B}_{R}(\chi)=\int_{\Gamma_{R}} \chi \boldsymbol{\psi} \boldsymbol{\psi}^{T}
$$

that represents the Robin b.c.. Using this notation, the Finite Element matrix associated to the bilinear form $a(\cdot, \cdot)$ in $(29)$ is given by

$$
\begin{aligned}
\mathbf{A}(\mathbf{K}, \mathbf{b}, c) & =\mathbf{R}_{x x}(\mathbf{K})+\mathbf{R}_{x y}(\mathbf{K})+\mathbf{R}_{y y}(\mathbf{K}) \\
& +\mathbf{R}_{x}(\mathbf{b})+\mathbf{R}_{y}(\mathbf{b})+\mathbf{R}(c)+\mathbf{B}_{R}(\chi) .
\end{aligned}
$$

We moreover define the vectors

$$
\left(\gamma_{N}\right)_{j}=\int_{\Gamma_{N}} \gamma_{N} \psi_{j}, \quad\left(\gamma_{R}\right)_{j}=\int_{\Gamma_{R}} \gamma_{R} \psi_{j} .
$$

The Finite Element solution $\hat{f}_{h}$, when the Dirichlet b.c. are homogeneous or when there are no Dirichlet b.c. is given by $\hat{f}_{h}=\boldsymbol{\psi}^{T} \hat{\mathbf{f}}$ where $\hat{\mathbf{f}}$ is the solution of the linear system

$$
\left[\begin{array}{cc}
\boldsymbol{\Psi}^{T} \boldsymbol{\Psi} & \lambda \mathbf{A}^{T} \\
\mathbf{A} & -\mathbf{R}
\end{array}\right]\left[\begin{array}{c}
\hat{\mathbf{f}} \\
\hat{\mathbf{g}}
\end{array}\right]=\left[\begin{array}{c}
\boldsymbol{\Psi}^{T} \mathbf{z} \\
\mathbf{u}+\mathbf{h}_{N}+\mathbf{h}_{R}
\end{array}\right]
$$

It should be noticed that the bilinear form in (29) differs from the one in (12) only for the term corresponding to Robin b.c., and the same of course holds for the Finite Element matrix (30) vs (14). All the non-homogeneous b.c. are instead included in the forcing term of the linear system. For this reason the smoothing matrix $\mathbf{S}$ depends only on the Robin b.c., while the vector $\mathbf{r}$ depends on all the non-homogeneous conditions.

If there are instead non-homogeneous Dirichlet conditions ( $\Gamma_{D}$ is non-empty and $\gamma_{D} \neq 0$ ) we need to define a so-called lifting of the boundary conditions. We consider in this case the space $V_{h}^{r}$ in (10) and denote by $\psi_{1}^{D}, \ldots, \psi_{N_{h}^{D}}^{D}$ the basis functions associated to the nodes $\boldsymbol{\xi}_{1}^{D}, \ldots \boldsymbol{\xi}_{N_{h}^{D}}^{D}$ on $\Gamma_{D}$. The problem is treated in this case by splitting the discrete surface estimator in two parts $f_{D, h}$ and $\hat{s}_{h}$, with $\hat{f}_{h}=f_{D, h}+\hat{s}_{h}$. The first part $f_{D, h} \in \operatorname{span}\left\{\psi_{1}^{D}, \ldots, \psi_{N_{h}^{D}}^{D}\right\}$ satisfies the non-homogeneous Dirichlet conditions on $\Gamma_{D}$, i.e., $f_{D, h}\left(\boldsymbol{\xi}_{i}^{D}\right)=\gamma_{D}\left(\boldsymbol{\xi}_{i}^{D}\right)$ for $i=1, \ldots, N_{h}^{D}$. The second part $\hat{s}_{h} \in V_{h, \Gamma_{D}}^{r}$ is instead the solution, with homogeneous Dirichlet b.c., of the variational problem:

$$
\left\{\begin{aligned}
a\left(\hat{s}_{h}, \psi_{h}\right) & -\int_{\Omega} \hat{r}_{h} \psi_{h}=\int_{\Omega} u \psi_{h}+\int_{\Gamma_{N}} \gamma_{N} \psi_{h} \\
& +\int_{\Gamma_{R}} \gamma_{R} \psi_{h}-a\left(f_{D, h}, \psi_{h}\right) \\
\lambda a\left(\varphi_{h}, \hat{r}_{h}\right) & +\sum_{i=1}^{n} \hat{s}_{h}\left(\mathbf{p}_{i}\right) \varphi_{h}\left(\mathbf{p}_{i}\right) \\
& =\sum_{i=1}^{n}\left(z_{i}-f_{D, h}\left(\mathbf{p}_{i}\right)\right) \varphi_{h}\left(\mathbf{p}_{i}\right)
\end{aligned}\right.
$$

for all $\psi_{h}, \varphi_{h} \in V_{h, \Gamma_{D}}^{r}$, where $\hat{r}_{h}$ is the adjoint variable associated to $\hat{s}_{h}$. This system has an extra forcing term, with respect to system (28), that implicitly involves the Dirichlet b.c. $\gamma_{D}$ through the quantity $f_{D, h}$.

Finally, in the areal data framework we can proceed analogously to prove the existence and the uniqueness of the surface estimator and to discretize the estimation problem. 